\title{
Therapeutic strategies to fight COVID-19: which is the status artis?
}

\author{
Cristina Scavone ${ }^{1}$, Annamaria Mascolo ${ }^{1}$, Concetta Rafaniello ${ }^{1}$, Liberata Sportiello ${ }^{1}$, Ugo \\ Trama $^{2}$, Alice Zoccoli ${ }^{3}$, Francesca Bernardi ${ }^{2}$, Giorgio Racagni ${ }^{4}$, Liberato Berrino ${ }^{5}$, \\ Giuseppe Castaldo ${ }^{6}$, Enrico Coscioni ${ }^{7}$, Francesco Rossi ${ }^{1}$, and Annalisa Capuano ${ }^{1}$ \\ ${ }^{1}$ Università degli Studi della Campania Luigi Vanvitelli \\ ${ }^{2}$ Regional Pharmaceutical Unit \\ ${ }^{3}$ Campus Bio-Medico University \\ ${ }^{4}$ Università degli Studi di Milano \\ ${ }^{5}$ University of Campania Luigi Vanvitelli \\ ${ }^{6}$ Federico II University Hospital \\ ${ }^{7}$ Agenzia Nazionale per i Servizi Sanitari Regionali
}

January 18, 2021

\begin{abstract}
COVID-19 is a complex disease and many difficulties are faced today especially in the proper choice of pharmacological treatments. The role of antiviral agents for COVID-19 is still being investigated. The evidence for immunomodulatory and antiinflammatory drugs is quite conflicting, while the use of corticosteroids is supported by robust evidence. The use of heparins in hospitalized critically ill patients is preferred over other anticoagulants. Lastly, conflicting data were found regarding to the use of convalescent plasma and vitamin D. According to data shared by the WHO, many vaccines are under phase 3 clinical trials and some of them already received the marketing approval in EU countries and in the US. In conclusion, drugs repurposing has represented the main approach recently used in the treatment of patients with COVID-19. At this moment, the analysis of efficacy and safety data of drugs and vaccines used in real life context is strongly needed.
\end{abstract}

\section{Introduction}

The vast majority of countries around the world are going through the second wave of COVID-19 pandemic, which constitutes an imminent threat to society, both in terms of human lives' loss and devastating economic consequences. Up to December $17^{\text {th }}$, more than $74,635,000$ people have contracted the disease and deaths exceed 1,657,000 (Worldometer, 2020). Since the beginning of the outbreak, the main features of SARS$\mathrm{CoV}-2$, the clinical characteristics of the disease and the role of many pharmacological treatments have been extensively studied. For instance, nowadays it is well known that the COVID-19 is a complex disease with several clinical phases of progression, affecting many organs apart from the respiratory tract. Specifically, its progression seems to follow four main stages (Cordon-Cardo et al., 2020). The first one is the phase during which the SARS-CoV-2 binds to epithelial cells and starts its replication. This stage occurs in the initial 1-2 days. The main proteins involved in the cell entry of SARS-CoV-2 are ACE2 receptor and TMPRSS2 (Fehr and Perlman, 2015; Hoffmann et al., 2020) and the soluble form of ACE2 receptor could be the target of potential therapeutic strategies (Scialo et al., 2020). Many patients can be asymptomatic at this stage and the innate immune response is commonly limited. In the second stage, the virus migrates down the respiratory tract, leading to the occurrence of many symptoms, such as fever, cough, shortness of breath, fatigue, muscle pain, headache, loss of taste or smell, sore throat, nausea or vomiting, and diarrhoea (Liu et al., 2020b). In this stage the innate immune response is triggered and an increase in the level of CXCL10 or other innate response cytokine (the so-called "cytokine storm") is observed as well (Wang et al., 2011; Qian et al., 2013). 
The third stage, which commonly occur within 1-2 weeks after symptom onset, is represented by the multisystem inflammation. Given the seriousness of symptoms (dyspnoea and hypoxia, ground glass infiltrate and progression to acute respiratory distress syndrome - ARDS), and possible cardiac, kidney, and liver damage), patients frequently require hospitalization (Guo et al., 2020a). This is also the phase in which abnormal coagulation biomarkers can be detected (such as, elevated D-dimer), representing sub-clinical progression toward stage 4. Lastly, a generalized hyper-inflammatory state is common in the stage (Del Valle et al., 2020; Leppkes et al., 2020). Lastly, a number of patients may reach the most critical and lethal stage (the last one), which is characterized by endothelial damage, thrombosis, and multi-organ dysfunction. The elevated levels of von Willebrand factor and endothelialitis together with hyperinflammation and hypercoagulability lead to microthrombi formation and systemic microvascular dysfunction (Nadkarni et al., 2020). On the other hand, the combinations of the severe respiratory failure and multi-organ failure, acute neurological disease, venous and arterial thromboembolic events, contribute to the increase in mortality for patients at this stage. In figure 1 the main COVID-19 symptoms by organs and tissues are shown. The clinical features of COVID19 and the inter-patients variability in its progression demonstrate the complexity of this disease but also the difficulties faced today in the proper choice of pharmacological treatments and patients' management. Several clinical trials and observational studies have been recently carried out worldwide. In the first wave of COVID-19 pandemic, many drugs have been used in the different phases of the disease. In fact, last May, we reported an overview of the benefit/risk profile of pharmacological treatments used in patients suffering from COVID-19 (Scavone et al., 2020). Considering new evidence recently acquired on the effect of different pharmacological treatments in patients with COVID-19, in this paper we aim to provide an up-to-date overview of medicines, including antivirals, antinflammatory and immune-modulating drugs, anticoagulants and other therapies which have been used around the world to treat COVID-19 and the related evidence in terms of efficacy and safety profile from interventional and observational clinical studies. Lastly, an update of vaccines under advanced clinical development is provided as well.

\section{Clinical evidence of antiviral agents}

A large number of antiviral agents, many of which are used for the treatment of other diseases, are currently undervaluation for COVID-19. The figure 2 shows the main drugs evaluated for COVID-19 and their mechanisms of action. We reported below those for which clinical evidence on their safety and efficacy is available.

Remdesivir is an antiviral drug effective against a broad spectrum of RNA viruses (including MERS-CoV and and SARS-CoV) (Agostini et al., 2018; de Wit et al., 2020; Sheahan et al., 2020). It is a nucleotide analogue that inhibits the RNA-dependent RNA polymerase (RdRp) essential for viral replication. A randomised, double-blind, placebo-controlled, multicenter trial, conducted on adults (aged [?]18 years) admitted to hospital with laboratory-confirmed SARS-CoV-2 infection, showed no difference for the time to clinical improvement between the remdesivir group and the placebo group (Wang et al., 2020b). A randomized, open-label trial, evaluating the efficacy of 5 or 10 days of remdesivir treatment compared to the standard care in hospitalized patients with confirmed severe acute respiratory syndrome and moderate COVID-19 pneumonia (pulmonary infiltrates and room-air oxygen saturation $>94 \%$ ), showed that patients treated with 10-day course of remdesivir had no statistically significant difference in clinical status (at 11 days) compared to standard care. On the contrary, those treated with 5-day course of remdesivir had a statistically significant difference in clinical status, but the difference was defined as uncertain for clinical importance (Spinner et al., 2020). A double-blind, randomized, placebo-controlled trial (NIAID-ACTT-1) of intravenous remdesivir administered to adults hospitalized for COVID-19 showed that remdesivir was superior to placebo in shortening the time to recovery. Specifically, patients receiving remdesivir had a median recovery time of 10 days (95\% confidence interval [CI], 9 to 11) compared with 15 days of patients receiving placebo (95\% CI, 13 to 18) (Beigel et al., 2020). Finally, a randomized, open-label, phase 3 trial on hospitalized patients with severe COVID-19 not requiring mechanical ventilation, which were randomized to receive intravenous remdesivir for 5 or 10 days, showed no difference in terms of clinical status between the two groups (Goldman et al., 2020). A meta-analysis of aforementioned clinical trials showed that the treatment with remdesivir for 10 days increased the recovery rate at day 14 in severe COVID-19 patients (RR=1.5, 95\% CI 1.33-1.7) 
and at day 28 in moderate and severe COVID-19 patients ( $\mathrm{RR}=1.14,95 \% \mathrm{CI} 1.06-1.22)$. Additionally, in all patients, remdesivir decreased the mortality rate at day 14 , but not at day 28 . The best response to remdesivir for both recovery and mortality rates was observed in nonmechanically ventilated COVID-19 patients at day 14 (Elsawah et al., 2020). Among observational data, a study conducted on patients under mechanical ventilation with confirmed COVID-19 showed a significant beneficial effect of remdesivir on survival (Pasquini et al., 2020). Moreover, a clinical improvement was also observed in hospitalized patients with severe COVID-19 who were treated with compassionate-use remdesivir (Grein et al., 2020). Based on the results of the NIAID-ACTT-1 trial, the European Medicine Agency (EMA) has granted a conditional marketing authorisation to remdesivir (Veklury ${ }^{\circledR}$ ) for the treatment of adults and adolescents from 12 years of age with COVID-19 pneumonia who require supplemental oxygen. Remdesivir is the first medicine that was recommended for COVID-19 in Europe with data evaluated in a short timeframe through a rolling review procedure, which is an approach used during public health emergencies to rapidly evaluate data. However, on November $20^{\text {th }}, 2020$ the World Health Organization (WHO) has issued a conditional recommendation against the use of remdesivir in hospitalized patients with COVID-19, regardless of disease severity, as there is no evidence demonstrating an improvement of survival or other outcomes during its use in these patients. This recommendation is based on the interim results of the open-label WHO Solidarity Trial and from data of 3 other randomized controlled trials, considering data from over 7000 patients across the 4 trials (World Health Organization). Currently, the Committee for Medicinal Products for Human Use (CHMP) of EMA is evaluating data on mortality at day 28 derived from the NIAID-ACTT-1 trial. Moreover, the EMA has requested the full Solidarity data from WHO and the marketing authorisation holder in order to assess, together with any other relevant available data, if any changes are needed to the marketing authorisation of remdesivir. In terms of safety, the EMA is also evaluating a signal for kidney toxicity, a condition that could have other causes in COVID-19 patients (European Medicine Agency).Lopinavir/ritonavir is a combination composed by lopinavir, a protease inhibitor with high specificity for HIV-1 and HIV-2, and ritonavir, an inhibitor of cytochrome P450 that allows to increase lopinavir plasma concentration (Scavone et al., 2020). First results from a randomized trials conducted in patients with SARS-CoV-2 infection did not show any benefit for this combination compared to the standard care alone (Cao et al., 2020; Li et al., 2020d), even though the triple antiviral therapy (lopinavir/ritonavir, ribavirin, and interferon beta-1b) was safer and more effective than lopinavir/ritonavir alone (Hung et al., 2020).In addition, the RECOVERY trial that aimed to compare a range of possible treatments with usual care in patients hospitalized for COVID-19 showed no reductions in 28-day mortality, duration of hospital stay, or risk of progressing to invasive mechanical ventilation or death for the lopinavir/ritonavir group (Horby et al., 2020b). A recent meta-analysis, comparing the treatment with lopinavir/ritonavir with the standard of care/control or any other antiviral agent/combinations, showed no difference between the lopinavir/ritonavir combination and the standard of care in terms of progression to more severe state, mortality, and virological cure on days 7-10. Moreover, no difference in efficacy was observed with lopinavir/ritonavir compared to umifenovir or hydroxychloroquine (Bhattacharyya et al., 2020). Another meta-analysis demonstrated no significant difference in terms of negative polymerase chain reaction (PCR) results between patients treated with lopinavir/ritonavir and those treated with the standard care (Wang et al., 2020a). Data from observational studies revealed no differences between lopinavir/ritonavir and hydroxychloroquine administered in patients with severe or mild-moderate COVID-19 (Choi et al., 2020; Karolyi et al., 2020; Lecronier et al., 2020). A retrospective study, conducted instead in non-severe patients with COVID-19, also showed no improvement in the prognosis or shortening of clinical course with lopinavir/ritonavir treatment (Gao et al., 2020). On the contrary, a retrospective cohort study showed that the combined antiviral therapy (lopinavir/ritonavir plus umifenovir) is more effective than lopinavir/ritonavir monotherapy (Deng et al., 2020). Based on the available evidence, the regular use of lopinavir/ritonavir in the treatment of COVID-19 cannot be supported. Some evidences suggest its use as a combination therapy but further clinical trials are needed to evaluate lopinavir/ritonavir's efficacy and safety in COVID-19 patients.

Favipiravir is a drug authorized for the treatment of influenza virus infections in Japan. It is a prodrug converted by intracellular phosphoribosylation into the active form able to inhibit the RdRp. Preliminary data suggested its safety and efficacy in shortening the time to recovery and in reducing pneumonia 
symptoms (Scavone et al., 2020; WATANABE et al., 2020). A randomized clinical trial, comparing the efficacy and safety of favipiravir vs umifenovir in hospitalized patients with COVID-19, demonstrated a higher efficacy for favipiravir than umifenovir $(\mathrm{p}=0.01)$. Regarding adverse events, liver enzyme abnormalities, psychiatric disorders, gastrointestinal symptoms, and serum uric acid elevations were those most commonly reported (Chen et al., 2020a). An open-label study, evaluating the effects of favipiravir versus lopinavir/ritonavir in patients with COVID-19 who were also treated with aerosol inhalation of interferon (IFN)- $\alpha$, showed a faster viral clearance and a better chest computed tomography change for the favipiravir group. Additionally, fewer adverse events were observed in the favipiravir group than lopinavir/ritonavir group (Cai et al., 2020). On the contrary, the combination favipiravir and inhaled IFN beta-1b showed no difference for inflammatory biomarkers at hospital discharge and for the overall length of hospital stay when compared to hydroxychloroquine(Khamis et al., 2020). Another study, aiming to evaluate the clinical outcomes and plasma concentrations of baloxavir marboxil and favipiravir in COVID-19 patients, failed in demonstrating a virological effect and clinical benefits of these two drugs. Authors concluded that this result could be determined by the insufficient concentrations of these drugs relative to their antiviral activities (Lou et al., 2020). An adaptive, multicenter, open label, randomized, phase II/III clinical trial of favipiravir versus standard of care in hospitalized patients with moderate COVID-19 pneumonia demonstrated a rapid antiviral response with favipiravir (Ivashchenko et al., 2020). Based on these preliminary results, the Russian Ministry of Health granted, in May 2020, a fast-track marketing authorization to favipiravir for the treatment of COVID-19 patients. A meta-analysis of some aforementioned clinical trials showed a significant clinical and radiological improvement with favipiravir compared to the standard of care, but no significant difference with regards to viral clearance, oxygen support requirement, or side effects (Shrestha et al., 2020). Currently, two clinical trials are ongoing to evaluate the efficacy and safety of favipiravir alone (NCT04336904) or in combination with tocilizumab (NCT04310228) for the treatment of COVID-19. The promising effectiveness of favipiravir for COVID-19 was also showed in a retrospective observational study (Rattanaumpawan et al., 2020). Based on the available evidence, favipiravir is effective in alleviate symptoms and in the clinical improvement of COVID-19 patients but further studies are need to prove its benefit in terms of viral clearance, oxygen support requirement, and mortality.Darunavir/cobicistat is another agent considered as a potential treatment for SARS-CoV-2 infection. Darunavir is an inhibitor of dimerization and of the HIV-1 protease, while cobicistat is an inhibitor of cytochromes P450 that increases darunavir plasma concentration (Deeks, 2018). Today, there are conflicting in vitro data on the effect of darunavir in inhibiting SARS-CoV-2 viral replication (Alshaeri and Natto, 2020; De Meyer et al., 2020). Results from a single-center, randomized, and open-label trial of darunavir/cobicistat plus interferon alpha $2 \mathrm{~b}$ vs interferon alpha $2 \mathrm{~b}$ alone in COVID-19 patients showed no difference in the proportion of negative PCR results at day 7 between the two groups (Chen et al., 2020b). Nicolini et al reported the results on the real-life use of darunavir/cobicistat in severe COVID-19 patients. Their findings showed that, although well tolerated, this treatment did not reduce mortality in COVID-19 (Nicolini et al., 2020). On the contrary, a case-control study showed a lower mortality for darunavir/cobicistat group than the control group (Odds Ratio, OR 0.07, 95\% CI 0.01-0.52, p=0.009) in critically ill patients with SARS-CoV-2 infection (Kim et al., 2020).Triazavirin, a guanosine nucleotide analogue that inhibits RNA synthesis, was compared to placebo in a pilot randomized controlled trial for the treatment of COVID-19. This study found no difference in the time to clinical improvement between groups in spite of a less frequent use of concomitant therapies for respiratory, cardiac, renal, hepatic, or coagulation supports in the triazavirin group (Wu et al., 2020). Currently, a randomized, phase $2 / 3$ clinical trial is evaluating the effects of triazavirin vs. placebo in inpatients with mild-moderate COVID-19 (the study will end at December 31, 2021) (NCT04581915).Umifenovir is a membrane hemagglutinin fusion inhibitor that blocks the attachment of viral envelope protein to host cells (Liu et al., 2020a). As mentioned above, some clinical trials failed to prove a clinical benefit with umifenovir compared to supportive care (Li et al., 2020d) or favipiravir (Chen et al., 2020a) in COVID-19 patients. However, an observational study suggested its superiority to lopinavir/ritonavir in shortening the duration of positive RNA in COVID-19 patients (Zhu et al., 2020). Clinical trials are ongoing to evaluate the efficacy and safety of umifenovir (NCT04260594) or umifenovir, oseltamivir, and lopinavir/ritonavir (NCT04255017) for the treatment of COVID-19.Danoprevir is a hepatitis C virus NS3 protease inhibitor 
that was firstly evaluated compared to lopinavir/ritonavir in COVID-19 patients. The study found that the group treated with danoprevir had a shorter mean time to achieve both negative nucleic acid testing and hospital stays than lopinavir/ritonavir group (Zhang et al., 2020c). Two clinical trials are ongoing to evaluate the efficacy and safety of danoprevir in combination with ritonavir (NCT04345276) or ritonavir plus interferon (NCT04291729).Sofosbuvir and daclatasvir are direct-acting antivirals that represent potential candidates for the treatment of COVID-19. A trial, evaluating the effectiveness of sofosbuvir and daclatasvir compared to ribavirin in patients with severe COVID-19, showed a relative risk of death of 0.17 (95\% CI 0.04-0.73, $\mathrm{P}=0.02$ ) for the sofosbuvir/daclatasvir group (Eslami et al., 2020). Similarly, an open-label, multicentre, randomized controlled clinical trial conducted in adults with moderate or severe COVID-19 showed that the group treated with sofosbuvir/daclatasvir plus standard care had a significant reduction of the duration of hospital stay compared with standard care alone (Sadeghi et al., 2020). A single-centre, randomized, controlled trial of adults with moderate COVID-19, comparing the treatment with sofosbuvir, daclatasvir, and ribavirin to the standard care, demonstrated instead no difference in terms of median duration of hospital stay, number of Intensive care Unit (ICU) admissions, and the number of deaths between groups, but the cumulative incidence of recovery was higher in the sofosbuvir/daclatasvir/ribavirin group (Abbaspour Kasgari et al., 2020). All studies highlight the need of further investigations in larger clinical trials.Nafamostat and camostat mesilate are inhibitors of TMPRSS211, a protease fundamental for the penetration of coronaviruses into the cell (Mascolo et al., 2020). First evidence showed a clinical improvement with the use of nafamostat and hydroxychloroquine, or nafamostat and favipiravir in severe COVID-19 patients (Doi et al., 2020; Iwasaka et al., 2020). Moreover, nafamostat was also effective in three cases of elderly patients with COVID-19 pneumonia. Both drugs are currently undervaluation in different clinical trials (www.clinicaltrials.gov).Ivermectin, a drug used for parasite infestations, has the ability to reduce, in vitro, the viral RNA of SARS-CoV-2 (Caly et al., 2020). Today, clinical observational data on the use of this medicine are conflicting. The ICON study, a chart review of consecutive patients hospitalized with confirmed COVID-19 treated with or without ivermectin $(200 \mu \mathrm{g} / \mathrm{kg}$ at day 1 and at the discretion of the physician at day 7), showed a lower mortality in the ivermectin group, but no difference was found for the extubation rates or length of stay (Rajter et al., 2020). Another retrospective study conducted in hospitalized patients with COVID showed that a single dose of ivermectin $(200 \mu \mathrm{g} / \mathrm{kg})$ did not improve clinical and microbiological outcomes of patients with severe COVID-19. However, as Authors stated, the drug was given at late stages of infection (median 12 days after the start of symptoms) and at a standard single dose that could be lower than the half maximal inhibitory concentration (IC50) needed for SARS-CoV-2 infection (Camprubí et al., 2020). Ivermectin is also undervaluation in different clinical trials (www.clinicaltrials.gov). Preliminary results from a randomized, controlled, phase 3, clinical trials, evaluating the recovery from COVID-19, showed the efficacy of the combination ivermectin and doxycycline compared to placebo (NCT04523831). Finally, monoclonal antibodies (figure 2), such as meplazumab ,REGN-COV2, and bamlanivimab, have been developed to prevent the viral attachment of SARS-CoV-2 to host cells.

Meplazumab is listed among antiviral agents because, based on its mechanism of action, it can control the virus replication. In fact, it is an inhibitor of CD147, a glycoprotein involved in the viral entry of SARS-CoV2 by interacting with the coronavirus S protein. CD147 also has a pro-inflammatory activity and regulates cytokine secretion and leukocytes chemotaxis during viral infections. Preliminary results showed that meplazumab compared to the control group was associated with a faster improvement of pneumonia (Bian et al., 2020). Two clinical trials are ongoing to evaluate the safety and efficacy of meplazumab in patients with COVID-19 (NCT04275245, NCT04586153).REGN-COV2 is a cocktail of two potent monoclonal antibodies (casirivimab and imdevimab) targeting the spike protein of SARS-CoV-2. First descriptive data showed that REGN-COV2 is able to reduce the viral load and the time to alleviate symptoms in non-hospitalized patients with COVID-19. Moreover, REGN-COV2 also showed positive trends in reducing medical visits (Regeneron Pharmaceuticals). Clinical trials are currently ongoing to evaluate its efficacy and safety for the treatment (NCT04425629, NCT04426695, NCT04381936) or prevention (NCT04452318) of COVID-19, and a clinical trial is ongoing to assess its safety, tolerability, pharmacokinetics, and immunogenicity in healthy adult volunteers (NCT04519437). On November 21 ${ }^{\text {st }}, 2020$ The U.S. Food and Drug Administration (FDA) granted an emergency authorization for casirivimab and imdevimab for the treatment of mild to moderate 
COVID-19 in adults and paediatric patients ([?] 12 years and weighing at least $40 \mathrm{Kg}$ ) with positive results of direct SARS-CoV-2 viral testing and who are at high risk for progressing to severe COVID-19. This approval based on data from a clinical trial demonstrating a reduction of COVID-19-related hospitalization or emergency room visits within 28 days after treatment in patients at high risk for disease progression (Food and Drug Administration).Bamlanivimab is a recombinant, human IgG1 monoclonal antibody (mAb) directed against the spike protein of SARS-CoV-2. Recently, a Phase 1 study of bamlanivimab in hospitalized patients with COVID-19 was successfully completed (NCT04411628) (Eli Lilly and Company), and an interim analysis of an ongoing phase 2 clinical trial in outpatients with recently diagnosed mild or moderate COVID-19 (BLAZE-1, NCT04427501) showed that one of three doses of bamlanivimab accelerates the natural decline in viral load over time (Chen et al., 2020c). Moreover, bamlanivimab has demonstrated to reduce hospitalizations and symptoms compared to placebo, with the most pronounced effects observed in high-risk patients (Chen et al., 2020c). Based on these results, on November $9^{\text {th }}, 2020$ bamlanivimab was authorized by the U.S. FDA, as emergency use, for the treatment of mild to moderate COVID-19 in adults and paediatric patients ([?] 12 years), who are at high risk for progressing to severe COVID-19 or hospitalization. Bamlanivimab is recommended to be administered as soon as possible after a positive COVID-19 test and within 10 days of symptom onset (Eli Lilly and Company). Currently, bamlanivimab is being evaluated in a phase 3 clinical trial assessing the prevention of COVID-19 in residents and staff at long-term care facilities (BLAZE-2, NCT04497987), and in the National Institutes of Health-led ACTIV-2 study in ambulatory COVID-19 patients (Eli Lilly and Company). Both bamlanivimab and REGN-COV2 are not authorized for use in hospitalized patients or in patients requiring oxygen therapy due to COVID-19 as they resulted most effective when given to a patient shortly after diagnosis, and in patients who have high viral load or who have not yet mounted their own immune response. Other drugs potential therapeutics candidates for COVID-19, not mentioned above, for which we have less evidence are: azidovudine, galidesivir, tenofovir, lepidasvir, velpatasvir (Alshaeri and Natto, 2020; Scavone et al., 2020). In conclusion, the role of antiviral agents for COVID-19 is still being investigated and the few data available does not allow making any conclusion. Remdesivir is the only one recommended in Europe for COVID-19 but it was also recently questioned for efficacy and safety and it is currently undervaluation by the EMA. Regarding monoclonal antibodies, such as bamlanivimab, which is indicated in patients at high risk of hospitalization, a question should be raised: "how to define who might be at risk of hospitalization, since individuals who go to hospital are frequently difficult to predict?". Moreover, the place in therapy of bamlanivimab and REGN-COV2 is tricky too; it would be interesting to understand which type of patients would benefit from these therapies.

Lastly, a new monoclonal antibody is currently under early stage of development in Italy at the MAD (Monoclonal Antibody Discovery) Lab of the Fondazione Toscana Life Sciences. This investigational therapy was obtained starting from convalescent plasma. At this moment, researchers found that the antibody is able to bind the spike protein and disable the virus. Thus, this therapy could serve both to prevent and treat Covid-19 (The Florentine, 2020).

\section{Clinical evidence of immunomodulatory and anti-inflammatory agents}

Since the beginning of the COVID-19 pandemic, the determining role of inflammatory cytokines in the worsening of clinical conditions has been identified. Indeed, one of the reasons underlying the occurrence of serious symptoms (figure 1) associated with the clinical progression of SARS-CoV-2 infection is represented by the increase in levels of pro-inflammatory cytokines, mainly interleukins, granulocyte-colony stimulating factor and TNF- $\alpha$. These molecules are responsible for the so-called cytokine storm that, in turn, induces ARDS, organ failure and sepsis (Guo et al., 2020b). Many drugs, acting through different mechanisms, are able to block or reduce the effects mediated by the cytokines storm (figure 2).

Tocilizumab was one of the most studied drugs able to reduce the hyperinflammatory state associated with COVID-19. It is a monoclonal antibody (figure 2), authorized for the treatment of many diseases, including rheumatoid arthritis, which is active against the interleukin-6 receptor, one of the key mediators of the inflammatory process (Scott, 2017). At the beginning of the pandemic, the drug was tested in China to reduce lung complications in 20 patients with severe SARS-CoV-2 infection, being associated with a re- 
duction of oxygen requirement, resolution of CT lesions, normalization of lymphocyte count, reduction of C-reactive protein levels and hospital discharge (Xu et al., 2020). Few months ago, many trials evaluating the effects of tocilizumab started in Italy, after the approval of the Italian Medicine Agency and the Spallanzani Ethics Committee. However, despite the encouraging preliminary results, the availability of new evidence from other ongoing clinical trials has marked a change of course. Indeed, the study carried out by the Local Health Unit-IRCCS of Reggio Emilia (which evaluated the efficacy of tocilizumab at an early stage in patients with COVID-19 pneumonia who did not require invasive or semi-invasive mechanical ventilation procedures invasive) was earlier stopped, after the enrolment of 126 patients. The reason for the early conclusion lies in the absence of proof of benefit in treated patients either in terms of worsening (access to the ICU) or survival (Italian Medicine Agency, 2020a). Similarly, the results of the COVACTA trial, who was ongoing when we published our first paper (Scavone et al., 2020), did not reveal a benefit of tocilizumab over placebo in patients with severe COVID-19 pneumonia. Indeed, as reported in a press document released by the pharmaceutical company, no difference in clinical status between patients treated with tocilizumab and placebo were found (primary endpoint). In addition, no differences between groups were found regarding to the percentage of patients who died by week four and in ventilator-free days (Roche, 2020). Despite the negative results of these trials, tocilizumab was associated with positive outcome in other clinical studies, both interventional and observational. For instance, the single arm trial TOVICID-19 evaluated the effects of tocilizumab in hospitalized patients with SARS-CoV-2 infection, oxygen saturation at rest in ambient air [?] 93\% or required oxygen support or mechanical ventilation either non-invasive or invasive. One hundred and eighty patients received a dose of $8 \mathrm{mg} / \mathrm{kg}$ up to a maximum of $800 \mathrm{mg}$ per dose and a second administration $12 \mathrm{~h}$ after the first one if respiratory function did not recover. The results revealed that the treatment with tocilizumab seems to reduce lethality at 30 days, although its impact at 14 days seems less relevant (Perrone et al., 2020). Lewis TC et al. reported the results of an observational cohort study of patients hospitalized at 3 hospitals within the NYU Langone Health system in New York who received tocilizumab 400-mg IV once in addition to standard of care or standard of care alone. Out of 3,580 patients, 497 were treated with tocilizumab. The propensity score analysis showed that a lower number of death occurred in tocilizumab-treated patients and that tocilizumab was associated with improved survival compared to controls $(\mathrm{p}>0.001)$. No difference were found between groups in term of adjusted time to hospital discharge, while patients treated with tocilizumab had longer adjusted ICU length of stay and a higher adjusted infection rate (both p >0.001) than controls (Lewis et al., 2020). Tleyjeh IM et al. carried out a systematic review and meta-analysis of 24 studies, including 5 randomized controlled trials and 19 observational studies (enrolling 1,325 and 10,021 patients, respectively). The results of this study demonstrated that tocilizumab is able to reduce the risk of mechanical ventilation in hospitalized COVID-19 patients but not the short-term mortality, at least according to the results of randomized controlled trials. On the contrary, data from observational studies suggested an association between tocilizumab and lower mortality. Authors suggested that many reasons could explain differences found for the effect on the mortality, including the enrolment of different patient populations, a different inflammatory status, and timing/dosing of tocilizumab. Furthermore, in order to be sufficiently powered to detect a difference in mortality between tocilizumab and control groups, the sample size of clinical trials should have been substantially higher. In terms of safety profile, no higher risk of infections or any other adverse events was found with tocilizumab use (Tleyjeh et al., 2020). Given the evidence currently available on the effects of tocilizumab in COVID19 patients no firm conclusion can be drawn. We still have to wait for the results of other studies, such those from the RECOVERY trial, which is currently evaluating the efficacy of tocilizumab and other drugs, including low-dose dexamethasone, colchicine, convalescent plasma, REGN-COV2 and aspirin (University of Oxford, 2020b). Furthermore, biochemical parameters like serum IL6 levels and TH17 cells may contribute to select subgroups of COVID-19 patients that would specifically benefit from the treatment with Tocilizumab, according to the view of a personalized therapy in such patients (Cacciapuoti et al., 2020). Conflicting results were also obtained for sarilumab (figure 2), a drug belonging to the same drug class of tocilizumab. In our first document (Scavone et al., 2020), we stated that three randomized controlled trials were underway. Nowadays, the results of one trial investigating the effects of sarilumab in severely or critically ill patients hospitalized with COVID-19 did not meet its primary and secondary endpoints (Sanofi, 
2020b). Other studies, mainly observational, that investigated the effects of sarilumab were carried out on a very limited number of patients. For instance, Benucci $M$ et al. evaluated the efficacy of sarilumab in 8 patients (mean age: 62 years) hospitalized at the San Giovanni di Dio Hospital (Florence, Italy). The drug was administered in a dose of $400 \mathrm{mg}$ in combination with hydroxychloroquine, azithromycin, darunavir, cobicistat and enoxaparin. Seven patients had an improvement of the Horovitz index (oxygenation expressed by an increased $\mathrm{SpO} 2 / \mathrm{FiO} 2$ ratio). A progressive reduction in the serum amyloid A and CRP inflammation parameters was observed and patients were discharge within 14 days of hospitalization (Benucci et al., 2020). Another study, carried out at the Fondazione Policlinico Universitario A. Gemelli-IRCCS (Italy), evaluated the effects of sarilumab in 53 patients (median age: 66 years) with severe COVID-19. Almost $70 \%$ of patients were also treated with darunavir/ritonavir, $24 \%$ with lopinavir/ritonavir, while the remaining patients did not receive antiviral treatment. Hydroxychloroquine, heparin and azithromycin were administered in 94,3\%, $74,9 \%$ and $54,7 \%$ of patients, respectively. Thirty-nine patients were treated in medical wards (the majority with a single infusion), while 14 in ICU (the majority with two infusions). Among patients who received the drug in the medical wards, at 19 days median follow-up, almost $90 \%$ significantly improved. Among patients who received sarilumab in ICU, almost $36 \%$ were still alive at the last follow-up. The overall mortality rate was 5,7\% (Gremese et al., 2020). A further retrospective study was carried out at the Azienda Ospedaliera dei Colli - Cotugno Hospital (South of Italy) on 15 patients (12 treated with a single dose and 3 with two infusions). All patients received hydroxychloroquine, lopinavir/ritonavir and heparin, while 11 patients were also treated with methylprednisolone. Improvements in respiratory parameters were observed in 10 patients after sarilumab administration. Five patients who received sarilumab died (Montesarchio et al., 2020). Overall, the majority of clinical studies carried out on sarilumab were observational and limited by a low number of patients. In addition, many other drugs were co-administered. Therefore, it is not easy to fully appreciate the positive effect deriving from sarilumab administration. Thus, also for this drug further clinical data are strongly needed.Chloroquine and hydroxychloroquine (figure 2) are noteworthy too. These are antimalarial drugs that have also been used for the treatment of different rheumatic diseases (Mascolo et al., 2018). Both drugs show many pharmacological effects, including the stabilization of the lysosomal membranes, the inhibition of prostaglandin synthesis, polymorphonuclear chemotaxis and phagocytosis and a possible interference with the production of interleukin 1 by monocytes and inhibition of the release of superoxide by neutrophils (Italian Medicine Agency). These drugs have demonstrated to be effective in inhibiting the replication of SARS-CoV-2 in experimental models (figure 2) (Italian Medicine Agency) and have been increasingly tested for COVID-19 in many clinical studies. Specifically, many studies, which are currently ongoing, are evaluating the effects of hydroxychloroquine as prophylactic agent (Lother et al., 2020; Niriella et al., 2020; Tirupakuzhi Vijayaraghavan et al., 2020). At the moment, only one randomized, double-blind, placebo-controlled study on the prophylactic role of hydroxychloroquine was concluded. The trial enrolled 821 subjects who had had family or occupational exposure with a subject with COVID-19. According to the study results, there was no difference in the incidence of new onset of COVID-19 between participants treated with hydroxychloroquine (11.8\%) and those who received placebo (14.3\%). Adverse events were more commonly observed with hydroxychloroquine than with placebo, although no serious adverse reactions were reported (Boulware et al., 2020). Many clinical trials evaluated the efficacy and safety of hydroxychloroquine, alone or in combination with azithromycin, as therapeutic agent. Gautret $\mathrm{P}$ et al. carried out a single-arm interventional study, which evaluated the effects of hydroxychloroquine (plus azithromycin) in 36 hospitalized patients with COVID-19. Authors reported that a significant reduction in viral load was found on day 6 in patients treated with hydroxychloroquine compared to controls. The addition of azithromycin to hydroxychloroquine therapy significantly contributed to the reduction of viral load (Gautret et al., 2020). Another study enrolled 11 hospitalized patients treated with hydroxychloroquine and azithromycin. After 5-6 days of starting treatment, the nasopharyngeal swab gave a positive result in 8/10 patients. These virological results are in contrast with those reported by Gautret et al. and raise doubts about the effectiveness of the drugs combination (Molina et al., 2020). A further study found no difference in viral load reduction and clinical outcomes between hydroxychloroquine and standard of care (CHEN et al., 2020). In addition, the results of the randomized clinical study by Tang et al., which was carried out on 150 hospitalized patients with COVID-19 (148 with mild to moderate disease and 2 with severe disease), 
did not show any difference on the viral clearance at 28 days. The only positive result came from a post hoc analysis in a subgroup of patients who did not take antivirals in which a greater effect on symptoms was observed (Tang et al., 2020). Similarly, the results of a randomized, double-blind, placebo-controlled clinical trial (carried out in 491 outpatients at an early stage of COVID-19) found no differences in symptom severity over 14 days between hydroxychloroquine and placebo (relative difference $12 \% ; \mathrm{p}=0.117$ ). (Skipper et al., 2020). Huang et al. conducted a randomized study that showed no significant differences up to 14 days, in terms of viral clearance and improvement on CT scan between chloroquine and lopinavir/ritonavir, but on day 14, $100 \%$ of patients treated with chloroquine were discharged from the hospital, compared with $50 \%$ in the lopinavir / ritonavir group. However, it should be underlined that patients in the chloroquine group were younger and they had started the treatment earlier (Huang et al., 2020). Lastly, the preliminary results of the SOLIDARITY study seem to suggest that hydroxychloroquine, the combination lopinavir / ritonavir and IFN-based regimens have little or no effect on mortality at 28 days or on hospital course (World Health Organization, 2020c). Alongside with data on the efficacy profile of chloroquine and hydroxychloroquine, many data on the safety profile of both drugs were collected as well. For example, a recent warning discouraged the friendly use of chloroquine in the COVID-19 disease due to the possible side effects (like a severe haemolytic crisis) in G6PD deficient males and in unaware carriers of the disease, that are estimated to be about 400 million worldwide (Capoluongo et al., 2020). Based on these, the main regulatory agencies have issued many recommendations for clinicians. For instance, the EMA drew the attention on risks of serious adverse reactions (including heart rhythm disturbances) (European Medicine Agency, 2020a), highlighting the need for prescribers to closely monitor patients treated with both drugs (European Medicine Agency, 2020b). In addition, the European agency recommended the use of these medicines only in clinical trials or in national emergency management programs in hospitalized patients under close monitoring (European Medicine agency, 2020). The FDA has also highlighted the need for patients' monitoring when treated with hydroxychloroquine, especially regarding to the risk of severe changes in heart rhythm (tachycardia, atrial fibrillation and torsades de pointes), observed in patients treated with these drugs often in combination with azithromycin and other drugs able to prolong the QT (US Food and Drug Administration, 2020a). The American agency also recommended the use of chloroquine and hydroxychloroquine only in the context of clinical trials and revoked, in June 2020, the previously granted Emergency Use Authorization (US Food and Drug Administration, 2020a). In May 2020, the World Health Organization (WHO) suspended the hydroxychloroquine treatment arm in the multicentre clinical trial SOLIDARITY (World Health Organization, 2020e). In June 2020, the hydroxychloroquine treatment arm was reopened for enrolments, only to be permanently closed in the same month (Italian Medicine Agency, 2020b). Lastly, on November 2020, the PRAC of the EMA recommended updating the product information for chloroquine or hydroxychloroquine due to the risk of psychiatric disorders and suicidal behaviour (European Medicine Agency, 2020d). In conclusion, data related to the efficacy and safety profile of antimalarial drugs are quite conflicting. We should wait for the results of clinical trials currently ongoing in COVID-19 patients (www.clinicaltrials.gov). At the time of the publication of our first paper (Scavone et al., 2020), the evidence on the efficacy of corticosteroids in patients with COVID-19 was quite conflicting. Nowadays, we know that these drugs, especially dexamethasone and methylprednisolone, could be considered one of the mainstays in the treatment of COVID-19, especially for patients with severe disease, to prevent its worsening and complications and to resolve its serious symptoms. As reported in figure 2, corticosteroids are associated with multiple mechanisms, including the increase in the gene transcription of anti-inflammatory cytokines, the decrease in gene transcription of pro-inflammatory cytokines, chemokines, and adhesion molecules and a reduction in inflammatory response. All these effects seem to be responsible of the counteraction of the cytokine storm caused by SARS-CoV-2 infection. First and foremost, the preliminary results of the RECOVERY trial, which were recently published on the NEJM, provided the most significant evidence supporting the use of dexamethasone. In this controlled, open-label trial, patients were randomized to receive oral or intravenous dexamethasone $6 \mathrm{mg} /$ day for up to 10 days $(n=2,104)$ or the usual care alone $(n=4,321)$. Within 28 days after randomization, $22.9 \%$ of patients treated with dexamethasone and $25.7 \%$ of patients in the usual care group died (age-adjusted rate ratio, $0.83 ; 95 \%$ confidence interval $[\mathrm{CI}], 0.75$ to $0.93 ; \mathrm{P}<0.001$ ). According to the level of respiratory support, the incidence of death was lower in dexamethasone group among patients receiving invasive mechanical 
ventilation or oxygen without invasive mechanical ventilation (Horby et al., 2020a). These results, together with those obtained from other randomized clinical studies (Angus et al., 2020; Dequin et al., 2020; Jeronimo et al., 2020; Tomazini et al., 2020), showed that dexamethasone could be considered a treatment option in hospitalized subjects with severe COVID-19 infection who require oxygen therapy, whether or not they have mechanical ventilation (invasive or not invasive). Based on the preliminary results of RECOVERY trial, on September 2020 the CHMP of the EMA concluded that dexamethasone can be considered a treatment option for patients who require oxygen therapy and the EMA is endorsing the use of this drug in adults and adolescents (from 12 years of age and weighing at least $40 \mathrm{~kg}$ ) who require supplemental oxygen therapy at a dose of 6 milligrams once a day for up to 10 days (European Medicine Agency, 2020c). Recently the WHO REACT Working Group carried out a meta-analysis of seven randomized clinical trials that evaluated the efficacy of corticosteroids (dexamethasone, hydrocortisone, or methylprednisolone) in 1,703 critically ill patients with COVID-19 on all-cause mortality at 28 days and the occurrence of serious adverse events. The results showed that, compared with usual care or placebo, the OR for the association with mortality was 0.64 $(\mathrm{p}<0.001)$ for dexamethasone, $0.69(\mathrm{p}=0.13)$ for hydrocortisone and $0.91(\mathrm{p}=0.87)$ for methylprednisolone. Sixty-four serious adverse events were observed among patients randomized to corticosteroids and eighty serious adverse events among patients randomized to usual care or placebo. Authors concluded that the administration of systemic corticosteroids, compared with usual care or placebo, was associated with lower 28-day all-cause mortality (Sterne et al., 2020). Lastly, several clinical trials are currently underway or in development to evaluate corticosteroids for the treatment of COVID-19 (www.clinicaltrials.gov). Similarly to corticosteroids, nonsteroidal anti-inflammatory drugs (NSAIDs) (figure 2) and paracetamol represent valuable therapeutic options in patients with COVID-19, especially for outpatients at early stage (not severe form), to treat fever and muscle pain. At the beginning of the outbreak, the role of NSAIDs was misjudged due to some concerns that arose from few studies (Capuano et al., 2020b). Specifically, in 2009 a group of researchers from the University of Rochester highlighted that NSAIDs might lower host defence following infection or vaccination (Bancos et al., 2009). On April 2019 the French regulatory agency reported the results of a survey carried out by the regional pharmacovigilance centres of Tours and Marseille in which serious infectious complications occurred in patients receiving NSAIDs, mainly ibuprofen and ketoprofen, used in the treatment of fever and pain (ANSM, 2019). On March 2020, the French Minister of Health recommended the use of paracetamol instead of ibuprofen or oral cortisone for the treatment of fever in patients with COVID-19. This statement was supported also by the French Authorities, which announced that NSAIDs might worsen clinical conditions of patients with COVID-19 (Le monde, 2020; Ministère de la Santé, 2020). Recently few clinical data have become available, mainly from observational studies, regarding to the use of NSAIDs in patients with COVID-19. For instance, Rinott E et al. carried out a retrospective cohort study on 403 patients with COVID-19. The authors evaluated the association between the use of ibuprofen, administered from a week before diagnosis of COVID-19 throughout the disease, and the mortality and the need for respiratory support. Of the entire cohort, 87 patients received ibuprofen and 316 did not. No significant difference was found between groups in terms of mortality and respiratory support. The comparison of ibuprofen users with paracetamol users did not reveal any difference in mortality rates or the need for respiratory support (Rinott et al., 2020). A prospective cohort study enrolled patients with COVID-19, who were interviewed about NSAID use and infection outcomes (such as death, admission, severity, time to clinical improvement, oxygen requirement and length of stay) through a telephone questionnaire. According to study's results, neither the acute use of ibuprofen nor chronic NSAID use were associated with a greater risk of mortality compared to non-use (adjusted HR: 0.632 [95\% CI 0.073-5.441; P = 0.6758 and adjusted HR 0.492 [95\% CI 0.178-1.362; P = 0.1721], respectively) (Abu Esba et al., 2020). Lastly, a further multicentre, observational study, which collected data related to 1222 patients with COVID-19 admitted to eight UK hospitals (of whom 54 were routinely prescribed NSAIDs prior to admission), found no evidence that routine NSAID use was associated with higher COVID-19 mortality (Bruce et al., 2020). Other drugs able to reduce the hyperinflammation are currently under evaluation in patients with COVID-19, including baricitinib, ruxolitinib, aviptadil and eculizumab. For instance, the effects ofbaricitinib , a Janus kinase (JAK) inhibitor authorized for the treatment of rheumatoid arthritis and able to suppress the cytokine storm and to interrupt the passage and intracellular assembly of SARS-CoV-2 into the target cells (Zhang et al., 
2020b), were evaluated in 12 patients with mild-moderate COVID-19 pneumonia. At week 1 and week 2, the drug demonstrated to improve the clinical and laboratory parameters and none of the patients required ICU support. Even though encouraging, this study enrolled a limited number of patients, had no randomization and no control group (Cantini et al., 2020a). The same research group carried out an observational, retrospective, multicentre study in hospitalized patients with moderate COVID-19 pneumonia to compare the 2-week effectiveness and safety of baricitinib and lopinavir/ritonavir $(\mathrm{n}=113)$ vs. hydroxychloroquine and lopinavir/ritonavir $(\mathrm{n}=78)$. The results showed that the fatality rate was significantly lower among patients treated with baricitinib (0\% vs. $6.4 \% ; \mathrm{p}=0.010)$. Similarly, ICU admission was significantly lower among patients treated with baricitinib (0.88\% vs. $17.9 \%)$ (Cantini et al., 2020b). The drug was also used in a patient with severe COVID-19 with insufficient response to lopinavir/ritonavir, hydroxychloroquine, azithromycin and sarilumab. Authors reported that after the administration of baricitinib, the patient experienced a prompt resolution of the respiratory function and improvement in the radiological findings (Cingolani et al., 2020). Based on data currently available, no firm conclusion can be drawn on the effects of baricitinib in COVID-19 patients. In addition, due to a possible increased risk of herpes zoster and simplex infections, a group of Italian researchers suggested that the use of baricitinib should be considered with extreme caution (Favalli et al., 2020). Ruxolitinib is a Janus kinase (JAK) 1 and JAK2 inhibitor authorized for the treatment of myeloproliferative malignancies (El Bairi et al., 2020). The effects of the drug were evaluated in the RUXCOVID study, a phase III, multicentre, randomised and placebo-controlled trial, but its results are still not available. Furthermore, the drug is currently evaluated in the RUXCOVID-DEVENT trial (NCT04377620) in COVID-19 patients with ARDS requiring mechanical ventilation.Aviptadil is a synthetic vasoactive intestinal polypeptide (VIP), which increases the activity of adenosine cyclase (Leuchte et al., 2008). By acting as a weak pulmonary vasodilator, the drug is able to improve the oxygenation in patients with chronic lung disease (Ferrara et al., 2005). Currently, many clinical studies are evaluating the effects of aviptadil as therapeutic or prophylactic agent. For instance, clinical trials NCT04311697 and NCT04360096 are investigating the effects of aviptadil on acute lung injury, ARDS, and dyspnoea associated with COVID19, while the phase I study NCT04536350 will evaluate the prophylactic effect of aviptadil for COVID-19 in 80 participants (at this moment all studies are still recruiting participants) (www.clinicaltrials.gov). Lastly, two recent studies highlighted the benefits deriving from the treatment with eculizumab (Annane et al., 2020; Laurence et al., 2020). This is a monoclonal antibody approved for the treatment of atypical haemolytic uremic syndrome, refractory generalized myasthenia gravis and neuromyelitis spectrum disorders. The drug is able to inhibit the terminal portion of the complement cascade involved in the inflammatory response (Gralinski et al., 2018). The study carried out by Laurence J et al. reported the experience of 3 critical COVID-19 patients who experienced a marked decline in D-dimers and neutrophil counts after eculizumab treatment (Laurence et al., 2020). The results of the proof-of-concept study carried out by Annane D et al. showed that, in a population of 80 patients with severe COVID-19 admitted to ICU, the estimated survival was $82.9 \%$ with eculizumab and $62.2 \%$ without eculizumab (log-rank test, $\mathrm{P}=0.04$ ). Based also on the positive results on rapid decrease in lactate, blood urea nitrogen, bilirubin levels and a rapid increase in platelet count and prothrombin time observed with eculizumab, authors suggested that the drug could improve survival and reduce hypoxia in patients with severe COVID-19 (Annane et al., 2020). In conclusion, many immunomodulatory and anti-inflammatory drugs have been tested in patients with COVID-19, but today the evidence is quite conflicting for most of them. In addition, many of the published studies are observational or suffer from many limitations, including the lack of a sample size calculation or control groups and the use of surrogate endpoints (viral load instead of mortality rate). At present, the highest number of concluded clinical studies was found for tocilizumab, hydroxychloroquine and corticosteroids. Among these drugs, only the use of corticosteroids seems to be supported by robust evidence, while data related to the efficacy and safety of tocilizumab and hydroxychloroquine are quite conflicting. Therefore, further data from randomized controlled trial or well-designed observational studies are strongly needed.

\section{Anticoagulant and antiplatelet drugs}

As shown in figure 1, among the most serious clinical complications of COVID-19, there is the onset of a coagulopathy that accompanied by an hyperinflammatory state (representing the so-called cytokine storm) 
and progression to multiple-organ dysfunction is a cause of death in COVID-19 patients along with respiratory failure (Asakura and Ogawa, 2020). Firstly, the state of hyperinflammation and hypercoagulability was identified as disseminated intravascular coagulation (DIC) (Marietta et al., 2020). However, it was then noticed that the pathophysiology of COVID-19-associated DIC is different from that of classic DIC (septic or traumatic DIC) (Asakura and Ogawa, 2020). In fact, in COVID-19 patients, the most common pattern of coagulopathy is characterized by increased levels of fibrinogen and D-dimer, a mild prolongation of PT/aPTT, and a mild thrombocytopenia, which can also be absent in some patient (American Society of Hematology, 2020a; Atallah et al., 2020). On the contrary, the classic DIC is characterized by severe thrombocytopenia, extend prolongation of PT and aPTT, high levels of D-dimer, and reduced levels of fibrinogen (American Society of Hematology, 2020a; Atallah et al., 2020).

The exact mechanisms contributing to coagulopathy in COVID-19 patients are not completely understood. In general, inflammation and coagulation are known to be linked by different molecular signals (Li and Ma, 2017; Levi et al., 2020). Pro-inflammatory mediators can stimulate the expression of intravascular tissue factor, leukocyte adhesion molecules, and plasminogen activator inhibitor-1 (PAI-1) (Gozzo et al., 2020). Moreover, inflammation can activate the coagulation cascade by overexpressing thrombin both systemically and locally in the lungs, leading to fibrin deposition and subsequent tissue damage (Li et al., 2020c). SARS-CoV-2 could also directly damage vascular endothelial cells through its bond to ACE2, which could represent the first injury triggering the abnormal coagulation ( $\mathrm{Li}$ et al., 2020a). However, some studies showed that ACE2 is expressed mainly on type II pneumocytes, and almost absent in endothelial cells (McGonagle et al., 2020). In this context, the generalized hypercoagulable state of COVID-19 patients could be due to the involvement of type II pneumocytes, the extensive pulmonary microvascular network, and the extensive hyperinflammatory state that is similar to the macrophage activation syndrome (Gozzo et al., 2020; McGonagle et al., 2020). Finally, the development of hypoxemia, secondary to the COVID-19-induced ARDS, might also activate the coagulation cascade and could contribute to endothelial dysfunction (McGonagle et al., 2020).

A better understanding of the thromboembolic risk in patients suffering from COVID-19 could help to optimize both diagnostic strategies and pharmacological management (Capuano et al., 2020a; Lodigiani et al., 2020). To date, the anticoagulation therapy is part of the therapeutic management of COVID-19 patients and some authors have suggested of using this strategy in patients with elevated D-dimer levels (American Association for Clinical Chemistry, 2020). In this regards, an observational study found that the in-hospital mortality in patients who required mechanical ventilation was lower for those treated with anticoagulation than those not receiving the anticoagulant treatment (Paranjpe et al., 2020). Undoubtedly heparins , either unfractionated or at low molecular weight (LMWH), for their ability of blocking or limiting the state of hypercoagulability, represent a good therapeutic option for anticoagulation in patients with COVID-19. Heparins other than having anticoagulant properties appear to mitigate the inflammatory state exercising non-anticoagulant mechanisms such as: inhibition of heparanase activity, (responsible for endothelial leakage); chemokines, and cytokines neutralisation; interference with leukocyte trafficking; neutralisation of extracellular cytotoxic histones, and reduction of viral cellular entry (Buijsers et al., 2020). Therefore, the benefit of using heparins could be related to the ability of blocking both coagulation and inflammation. Accordingly, a retrospective observational study, evaluating the effect of LMWH treatment on disease progression, demonstrated that this treatment improved the coagulation dysfunction of COVID-19 patients and exerted anti-inflammatory effects by reducing IL-6 and increasing lymphocyte percentage (SHI et al., 2020). Another observational study found that the treatment with heparin was associated with a lower mortality in hospitalized patients with COVID-19 (Ayerbe et al., 2020). The WHO has recommended the use of LMWH, such as enoxaparin, in patients (adults and adolescents) hospitalized with COVID-19 to prevent venous thromboembolism, unless contraindicated (World Health Organization, 2020a). However, an argument debated is the optimal anticoagulant dosage that must be used in patients with COVID-19. A study suggested to strictly apply pharmacological thrombosis prophylaxis in all COVID-19 patients admitted to ICU, and to use high-prophylactic doses (Klok et al., 2020). To date, guidelines recommend to use prophylactic doses of LMWH in hospitalized COVID-19 patients unless contraindicated, but not in non-hospitalized patients (Gozzo et al., 2020; NIH, 2020; World Health Organization, 2020a). As reported by the American Society 
of Haematology, many protocols have adopted an intermediate-intensity dose (administering the usual daily LMWH dose twice daily) or even a therapeutic-intensity dose strategy for thromboprophylaxis based on local experience (American Society of Hematology, 2020b). Another guideline suggests to use in acutely/critically ill patients with COVID-19 a standard dose of anticoagulant over intermediate (LMWH twice-daily or increased weight-based dosing) or full treatment dosing (Moores et al., 2020). This recommendation is based on the lack of evidence regarding the risk of venous thromboembolism in hospitalized COVID-19 patients and the risk of bleeding in critically ill COVID-19 patients, who might also be at high risk of bleedings considering the severity of illness (Moores et al., 2020). Moreover, this guideline also suggests to prefer unfractionated heparins over LMWH in patients at high risk of bleeding (including those with severe renal failure) (Moores et al., 2020). A recent retrospective observational study, evaluating the impact of different doses of LMWH on the incidence of bleedings in COVID-19 patients admitted to ICUs, showed that the use of therapeutic doses of heparin did not increase the risk of bleeding in their patient population. Moreover, the study suggested the importance of applying a risk stratification based on D-dimer values for critically ill patients with COVID-19 (Pavoni et al., 2020). Based on these considerations, a close clinical monitoring and an individual patient evaluation for the risk of thrombosis and bleedings should be applied (Gozzo et al., 2020). Clinical trials are strongly needed to assess the optimal dose of heparin for COVID-19 and to assess their benefit/risk profile. Currently, different clinical trials are ongoing to evaluate the treatment with heparin in hospitalized patients with COVID-19 (www.clinicaltrials.gov). In Italy, for example, there are 5 ongoing clinical trials of patients with COVID-19 evaluating the efficacy of heparins (at different doses and regimen), alone or in combination with corticosteroids.

Among other pharmacological strategies that are being investigated for the prevention of thrombosis in COVID-19 patients, there isaspirin (acetylsalicylic acid ), an irreversible platelet inhibitor used for conditions such as myocardial infarction, strokes and pre-eclampsia in pregnant women. Aspirin, in addition to its anti-inflammatory and antithrombotic effects, has shown a significant antiviral activity against DNA and RNA viruses, including different human coronaviruses (Bianconi et al., 2020). Moreover, the use of aspirin has been associated with reduced thrombo-inflammation and lower rates of clinical complications and in-hospital mortality in different types of infections (Bianconi et al., 2020). Conflicting data have instead been reported on the use of aspirin in ARDS (Erlich et al., 2011; Kor et al., 2011, 2016; Boyle et al., 2015; Chen et al., 2015). Observational studies demonstrated some benefit in reducing the risk of ICU mortality, acute lung injury (ALI), and ARDS (Erlich et al., 2011; Boyle et al., 2015; Chen et al., 2015), while a larger observational study did not demonstrate any difference between aspirin use and ALI (Kor et al., 2011). Moreover, a randomized, double-blind, placebo-controlled, randomized clinical trial, conducted on 390 patients, showed that the use of aspirin compared with placebo did not reduce the risk of ARDS (Kor et al., 2016). First data from a retrospective, observational cohort study of adult patients hospitalized with COVID-19 have showed, after adjustment, that aspirin was associated with a decreased risk of mechanical ventilation, ICU admission, and in-hospital mortality, with no difference for major bleedings between aspirin and non-aspirin users (Chow et al., 2020). The drug will be tested for its effect of reducing blood clots in the world's largest clinical trial of treatments for patients hospitalised with COVID-19 (RECOVERY trial) (University of Oxford, 2020a). If effective, aspirin may be beneficial because it is safe, accessible and inexpensive.

Other than the aforementioned drugs, oral anticoagulants or other antiplatelet agents (P2Y12 receptor antagonists) are available in the market. However, concerns were raised about their interactions with multiple medications that are being used and tested for the treatment of COVID-19 (Gozzo et al., 2020). For instance, sarilumab and tocilizumab can reduce plasma concentrations of apixaban, rivaroxaban, and warfarin, while atazanavir and lopinavir/ritonavir can increase drug concentrations of apixaban and rivaroxaban and reduce the active metabolite of clopidogrel and prasugrel (American Society of Hematology, 2020b). Another question is about patients who are already in treatment with oral anticoagulants or antiplatelet agents for underlying conditions, for which guidelines suggest to continue their treatment even after the diagnosis of COVID-19 (NIH, 2020). In conclusion, the use of heparins in hospitalized critically ill patients is preferred over other anticoagulants because of the shorter half-life and fewer drug-drug interactions, being also the first choice for pregnant women. 


\section{Other therapies}

The use of convalescent plasma therapy in patients with COVID-19 is still being debated. Convalescent plasma is a mixture of inorganic and organic compounds, water and proteins (including albumin, immunoglobulins, complement, coagulation and antithrombotic factors) (Rojas et al., 2020). This treatment was formerly evaluated in the treatment of SARS (Cheng et al., 2005), MERS (Arabi et al., 2016) and Ebola (Kraft et al., 2015), being associated with a reduction in fatality rate. For COVID-19, stringent criteria need to be satisfied in order to be a convalescent donor. Specifically, donors should have the following characteristics: aged between 18 and 65 years; absence of infectious symptomatology; a negative test for COVID-19 after 14 days of recovery (which must be repeated $48 \mathrm{~h}$ later and at the moment of donation) (Tiberghien et al., 2020). The main procedure to obtain plasma is apheresis, which is based on a continuous centrifugation of blood from donor. From a single apheresis $400-800 \mathrm{~mL}$ of plasma can be obtained (Bloch et al., 2020). Nowadays, few clinical studies were concluded and their results could provide new insights regarding to the effectiveness and safety profile of plasma therapy in COVID-19 patients. Li 1 et al. reported the results of an open-label, multicentre, randomized clinical trial carried out in Wuhan, from February to April 2020. The trial enrolled 103 participants (median age: 70 years) with a severe form of COVID-19, who were randomized to receive convalescent plasma in addition to standard treatment $(\mathrm{n}=52)$ or the standard treatment alone $(\mathrm{n}=51)$. The effects of both therapies were evaluated as the time to clinical improvement within 28 days (primary outcome) and on 28-day mortality, time to discharge, and the rate of viral PCR results turned from positive at baseline to negative at up to 72 hours (secondary outcomes). According to the study's results, the convalescent plasma therapy was not associated with a statistically significant improvement in time to clinical improvement within 28 days compared with standard treatment alone. In addition, 2 patients in the convalescent plasma group experienced adverse events within hours after transfusion. Due to a decrease in the number of COVID-19 cases in late March, the study was stopped earlier; this needs to be take in consideration when the study's results are interpreted (Li et al., 2020b). Simonovich VA et al. carried out a randomized controlled trial in 334 hospitalized adult patients with severe COVID-19 pneumonia, who were randomized to receive convalescent plasma $(n=228)$ or placebo $(n=105)$ in addition to standard treatment. Authors evaluated the effects of the therapy in terms of changes in patient's clinical status 30 days after the intervention. Similarly to the previous study, no difference in terms of efficacy was found between treatments groups at day 30. Regarding to the safety profile, infusion-related adverse events were detected in $4.8 \%$ of patients in the convalescent plasma group vs. $1.9 \%$ of patients in the placebo group (odds ratio, 2.62; $95 \%$ CI, 0.57 to 12.04), even though no significant differences were found in the overall incidence of adverse events or serious adverse events (Simonovich et al., 2020). On the other hand, few small trial or case series reported that the convalescent plasma therapy might be beneficial in COVID-19 patients. For instance, Duan K et al. reported that the administration of $200 \mathrm{~mL}$ of convalescent plasma in 10 severe patients brought to a significant improvement in clinical symptoms along with increase of oxyhemoglobin saturation within 3 days and no severe adverse effects were observed (Duan et al., 2020). Zhang B et al. presented four critically ill patients with SARS-CoV-2 infection (including a 31-year-old pregnant woman) who received convalescent plasma and recovered from SARS-CoV-2 infection eventually and did not experience any serious adverse event (Zhang et al., 2020a). Lastly, a further case series described the effects of the convalescent plasma therapy in 5 critically ill patients with COVID-19 and ARDS, who improved after receiving the therapy (Shen et al., 2020). Despite their positive results, these studies' findings (Duan et al., 2020; Shen et al., 2020; Zhang et al., 2020a) should be interpreted with caution considering the limited number of patients who were enrolled and the studies' designs that did not allow making any comparison. In conclusion, based on the conflicting evidence currently available, the use of convalescent plasma needs to be considered as investigational. Regulatory agencies, such as the FDA, are releasing many documents in order to guide healthcare providers and investigators in the appropriate management of plasma therapy in the context of clinical trials (US Food and Drug Administration, 2020b).

Many studies are currently investigating the effects of vitamin D in COVID-19 patients (D'avolio et al., 2020). This interest derives from the evidence suggesting the role of vitamin $D$ in reducing the risk of cold and acute respiratory infections (Zemb et al., 2020). Many mechanisms seem to underline this effect, including the 
effects of Vitamin D on cellular natural immunity and adaptive immunity through the decrease in cytokine storm (this effect was observed on interferon $\gamma$, tumor necrosis factor $\alpha$ and CD4+ T cell count (Ali, 2020). In addition, vitamin $\mathrm{D}$ improves the production of antimicrobial peptides in the respiratory epithelium, potentially reducing the risk of local infection and it seems to interact with ACE2 (Mitchell, 2020). Pizzini A et al. investigated the association between of Vitamin D status and COVID-19 presentation among 109 patients, using data from the CovILD registry. They found that low Vitamin D levels were not associated with poor clinical and radiological outcomes of COVID-19 (Pizzini et al., 2020). A further study, which was based on data from the UK Biobank, aimed to establish whether blood 25-hydroxyvitamin D concentration was associated with COVID-19 risk or not. Data were available for 348,598 UK Biobank participants, of which 449 had COVID-19 infection. Authors concluded that no association was found in terms of potential association between vitamin D concentrations and the risk of COVID-19 infection (Hastie et al., 2020). Ultimately, current evidence on a potential correlation between Vitamin D and COVID-19 is contradictory. Therefore, we should wait for results from clinical trials, which are currently underway to evaluate the effects of Vitamin D supplementation on mortality, morbidity, prevention and treatment of COVID-19 (www.clinicaltrials.gov). Currently, data on vitamins, mineral supplementation and nutraceuticals are quite scarce, although some studies are investigating their effects (Jovic et al., 2020).

\section{Vaccines under advanced stage of clinical development}

According to the WHO, up to December $10^{\text {th }} 2020,52$ candidate vaccines are under clinical evaluation and 162 under preclinical evaluation (World Health Organization, 2020b). Specifically, 13 vaccines are already in advanced stage of clinical development, being evaluated in phase 3 clinical trials.

In table 1 an overview of these vaccines is reported. For some of these products, many clinical studies are currently undergoing but others have already provided preliminary results. Among these 13 vaccines, four are currently undervaluation by the EMA: ChAdOx1-S (AZD1222, Astrazeneca), LNP-encapsulated mRNA (mRNA-1273, Moderna), 3 LNP-mRNAs (BNT162b2, Pfizer), and Adenovirus Type 26 vector (Ad26.COV2.S, Janssen). Hereafter the preliminary efficacy and safety results for those vaccines are reported.

Indeed, on December 8th, the preliminary results of a phase 3 trials of the COVID-19 vaccine developed by AstraZeneca and Oxford University (ChAdOx1 nCoV-19 vaccine) were published in the Lancet journal. This vaccine was developed using a chimpanzee adenovirus viral vector that triggers the expression of the spike protein of SARS-CoV-2. Once injected, the human cells produce the protein and train the immune system to antibodies and T-cells against it. The published preliminary results refer to data from 4 ongoing randomised, controlled trials which are currently underway across the UK, Brazil, and South Africa (studies ISRCTN89951424, NCT04324606, NCT04400838, and NCT04444674). Adult participants were randomized to receive two doses of the vaccine [(both containing $5 \times 10^{1} 0$ viral particles - standard dose cohort (SD) or a low dose as first dose and a standard dose as second dose (LD/SD cohort)] or control (meningococcal group A, C, W, and Y conjugate vaccine or saline). From April until November 2020, 23,848 participants were enrolled. Of these, 11,636 participants were included in the interim primary efficacy analysis. In SD and $\mathrm{LD} / \mathrm{SD}$ cohort, the vaccine efficacy was $62,1 \%$ and $90 \%$, respectively. Starting from 21 days after the first dose, ten cases hospitalised for COVID-19 all in the control arm occurred. One hundred and seventyfive severe adverse events occurred, of which 84 in the ChAdOx1 nCoV-19 group and 91 in the control group. Among serious adverse events, there were cases of haemolytic anaemia and transverse myelitis. Authors concluded that the new vaccine is efficacious and it could contribute to control of the disease in this pandemic (Voysey et al., 2020). The British and Indian regulatory agencies recently granted the marketing approval for the Oxford University/AstraZeneca vaccine. Indeed, the UK MHRA provided authorization for emergency supply of this vaccine for the active immunization of subjects 18 years or older (AstraZeneca, 2020).

Pfizer developed a lipid nanoparticle-formulated, nucleoside-modified RNA vaccine that encodes a prefusion stabilized, membrane-anchored SARS-CoV-2 full-length spike protein, BNT162b2, whose efficacy and safety were recently published on the NEJM (Polack et al., 2020). Specifically, a multinational, placebo-controlled, 
pivotal efficacy trial, which is still ongoing, enrolled 43,548 patients aged $>16$ years of age and who were randomized to receive two doses, 21 days apart, of placebo or the BNT162b2 vaccine (30 $\mu \mathrm{g}$ per dose). A total of 43,448 received injections: 21,720 with the vaccine and 21,728 with placebo. Study's results revealed for the vaccine an efficacy in preventing COVID-19 equal to 95\% (95\% credible interval, 90.3 to 97.6); indeed, after the second dose, 8 cases of Covid-19 were detected among subjects who received BNT162b2 vs. 162 among those assigned to placebo. Similar results were found across subjects' subgroups by age, sex, race, ethnicity, baseline body-mass index, and the comorbidities. After the first dose, 9 cases of severe COVID-19 were detected among subjects receiving placebo vs. 1 case in those receiving BNT162b2. BNT162b2 demonstrated a good safety profile, being associated with short-term, mild-to-moderate injection site reactions, fatigue, and headache and no difference were detected between groups in term of serious adverse events (Polack et al., 2020). This vaccine was firstly approved in the UK with its administration started in December 2020, then it was authorized, during the same month, by the FDA for emergency use in subjects 16 years of age and older. Finally, on December $21^{\text {st }}, 2020$ the EMA has granted a conditional marketing authorisation for this vaccine to prevent COVID-19 in people from 16 years of age.

Moderna also developed a RNA-vaccine and recently shared data on the achievement of its study's primary efficacy endpoints. Indeed, on November $30^{\text {th }} 2020$, Moderna announced the results related to primary efficacy analysis of the COVE study (NCT04470427), a phase 3, randomized, placebo-controlled study, which is investigating the efficacy, safety and immunogenicity of mRNA-1273 SARS-CoV-2 vaccine in 30,000 adult subjects in the U.S (Moderna, 2020). The results of this trial, which were recently published on the NEJM, demonstrated that the mRNA-1273 vaccine has $94.1 \%$ efficacy at preventing Covid-19. Thirty subjects, all receiving placebo, developed severe Covid-19. Local and systemic reactions occurred in subjects who received the vaccine, but no safety concerns were identified. Serious adverse events were rare, and no differences were found between the placebo and the vaccine groups (Baden et al., 2020). On December $18^{\text {th }}$, 2020, the U.S. FDA issued an emergency use authorization for the second vaccine to prevent COVID-19 in individuals 18 years of age and older.

Lastly, the efficacy and safety of Adenovirus Type 26 vector (Ad26.COV2.S, Janssen - Johnson \& Johnson) is currently evaluated in phase 3 ENSEMBLE and ENSEMBLE 2, randomized, double-blind, placebo-controlled clinical trials (both studies will enroll approximately 90,000 subjects). On November $15^{\text {th }} 2020$, preliminary results from a phase $1 / 2$ a clinical study were published, showing that, after a single-dose among adult and elderly subjects, the vaccine induced a prompt and robust immune response (Janssen, 2020).

Lastly, many other vaccines are under evaluation in phase 1-2 clinical trials. Some of them are facing delays in achieving study's outcomes. For instance, Sanofi and GSK announced a delay in their adjuvanted recombinant protein-based COVID-19 vaccine program due to a low immune response in adults aged $>49$ years for an insufficient antigen concentration. The pharmaceutical companies state that they will carry out a Phase $2 \mathrm{~b}$ study with an improved antigen formulation (Sanofi, 2020a).

In conclusion, vaccination has already started in some countries with the Pfizer's vaccine administered in UK and US since December 2020, the Oxford University/AstraZeneca vaccine which was administered for the first time in the UK on January $4^{\text {th }} 2021$, the Chine's vaccine (CoronaVac) administered to Chinese since July, or the Gamaleya Research Institute's vaccine (Sputnik V) approved in Russia for emergency use since august 2020. Therefore, preliminary efficacy and safety data from real life will be soon available.

\section{Conclusion}

The spread of the new COVID-19 was inevitably followed by the research for therapies able to counteract severe signs and symptoms of this disease. Today, pharmacological researches are focusing on different drug classes, including antivirals, immunomodulatory and anti-inflammatory agents, anticoagulants and antiplatelet drugs, convalescent plasma, and vitamins. Other drugs are currently administered among inpatients and outpatients with Covid-19, such as antibiotics. The use of these drugs is, on many occasions, necessary, given that patients with COVID-19 may also develop bacterial infections, such as pneumonia. In this regard, it should be underline how much important is that the use of any antibiotic is under clinicians' strict control, 
due to the widespread and inappropriate use of these drugs even during the COVID-19 pandemic. Indeed, data recently shared by the WHO revealed that, while up to $15 \%$ of severe COVID-19 patients develop bacterial co-infection, almost $75 \%$ of patients actually receive them (World Health Organization, 2020d).

Given the absence of a specific drug able to block the replication of SARS-Cov-2, drugs repurposing has represented the main approach recently used. Indeed, many drugs that are currently used to neutralize COVID-19 signs and symptoms were already approved but for other therapeutic indications. This is the case, for example, of antivirals, whose role however is still debated. Also for remdesivir, which is the only drug recommended for COVID-19, some concerns related to its efficacy profile were raised by the WHO based on the results of open-label SOLIDARITY trial. Given these concerns, the EMA is currently re-evaluating the drug.

Many immunomodulatory and anti-inflammatory drugs have been tested in patients with COVID-19 as well. Based on current evidence and considering the limitations of published clinical studies on these drugs, no firm conclusion can be drawn. Among these drug classes, tocilizumab, hydroxychloroquine and corticosteroids have been extensively studied, even though only the use of corticosteroids seems to be supported by robust evidence, both for outpatients and inpatients requiring supplemental oxygen. In addition, it should be underline the significant role of NSAIDs, mainly ibuprofen and paracetamol, especially in patients suffering from a mild form of COVID-19 (early stage - manageable at home) to solve symptoms like fever and joint and muscle pain.

The role of heparins is noteworthy too. Indeed, the administration of these drugs in critically ill patients is crucial in order to reduce the thromboembolic risk, which is one of the most serious consequences of COVID-19.

In addition, based on the results of published studies, it is still not clear the role of convalescent plasma and vitamins. Therefore, we should wait for results from clinical trials, which are currently ongoing to evaluate the effects of these therapies on mortality, morbidity, prevention and treatment of COVID-19.

Other drugs, such as the combination of monoclonal antibodies REGN-COV2, might represent a powerful strategy to avoid patients' hospitalization and alleviate the burden on the health care system. At this moment, REGN-COV2 and bamlanivimab - which will be used for the treatment of mild to moderate COVID-19 in adult and pediatric patients - received the approval from the FDA, but not yet across European countries (Food and Drug Administration).

Lastly, out of 13 vaccines currently under phase 3 clinical development, four are under evaluation by the EMA. Among them, two are in the first rolling review evaluation, while the remaining are in the second phase of rolling review. The preliminary efficacy data published in the literature (for one vaccine) and through pharmaceutical industries' press releases (three vaccines) revealed a high efficacy rate and a good safety profile. However, according to the EMA, after the approval of these vaccines, further post-marketing studies that evaluate vaccine effectiveness are needed in order to better understand immediate and longerterm protection in a larger range of subjects. In conclusion, the analysis of real world data is strongly needed to confirm the favourable efficacy and safety profile of drugs currently used for the treatment of COVID-19 as well as of vaccines for its prevention.

Competing Interests' Statement: none. 


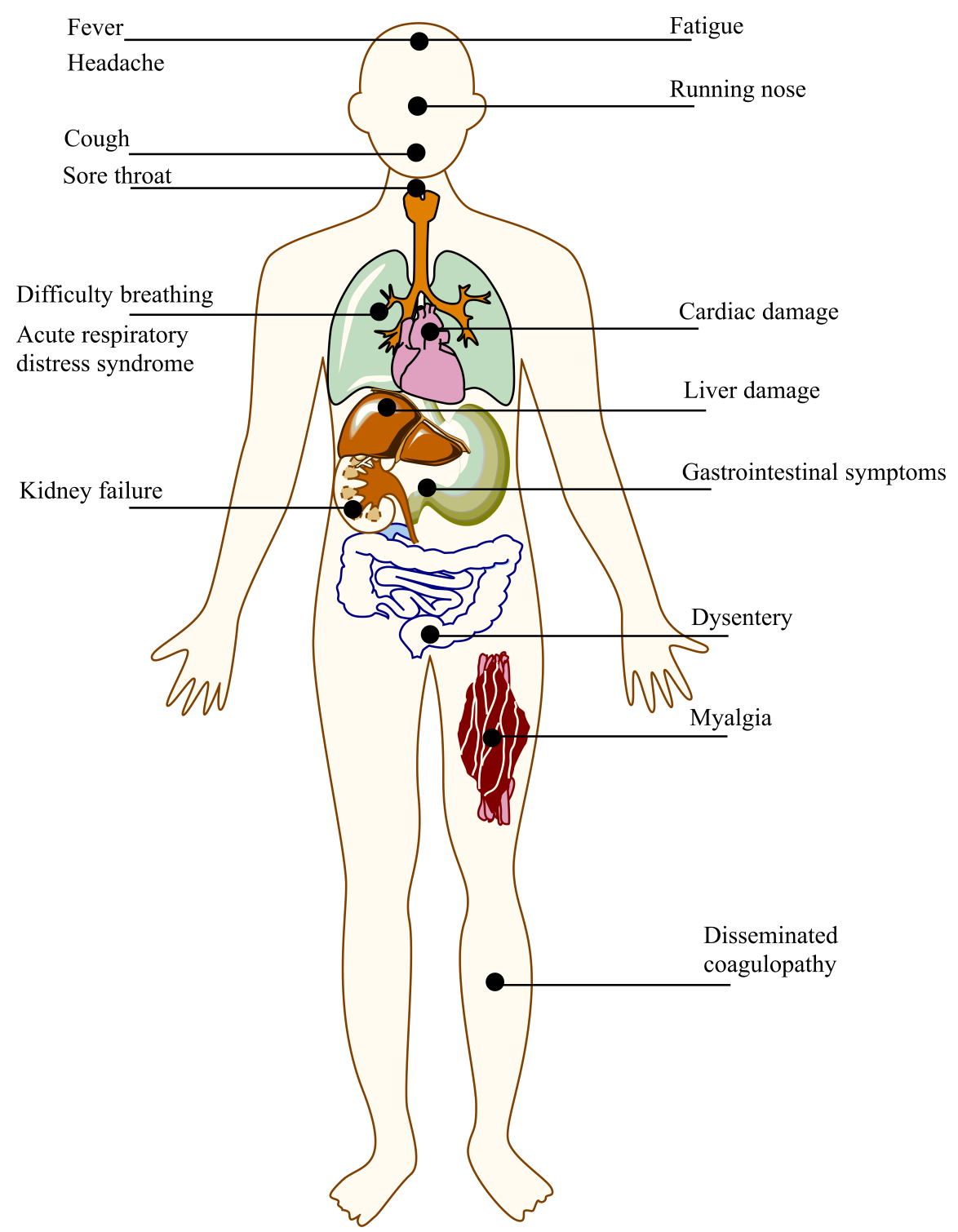

Figure 1. The main symptoms by organs and tissues related to COVID-19. 


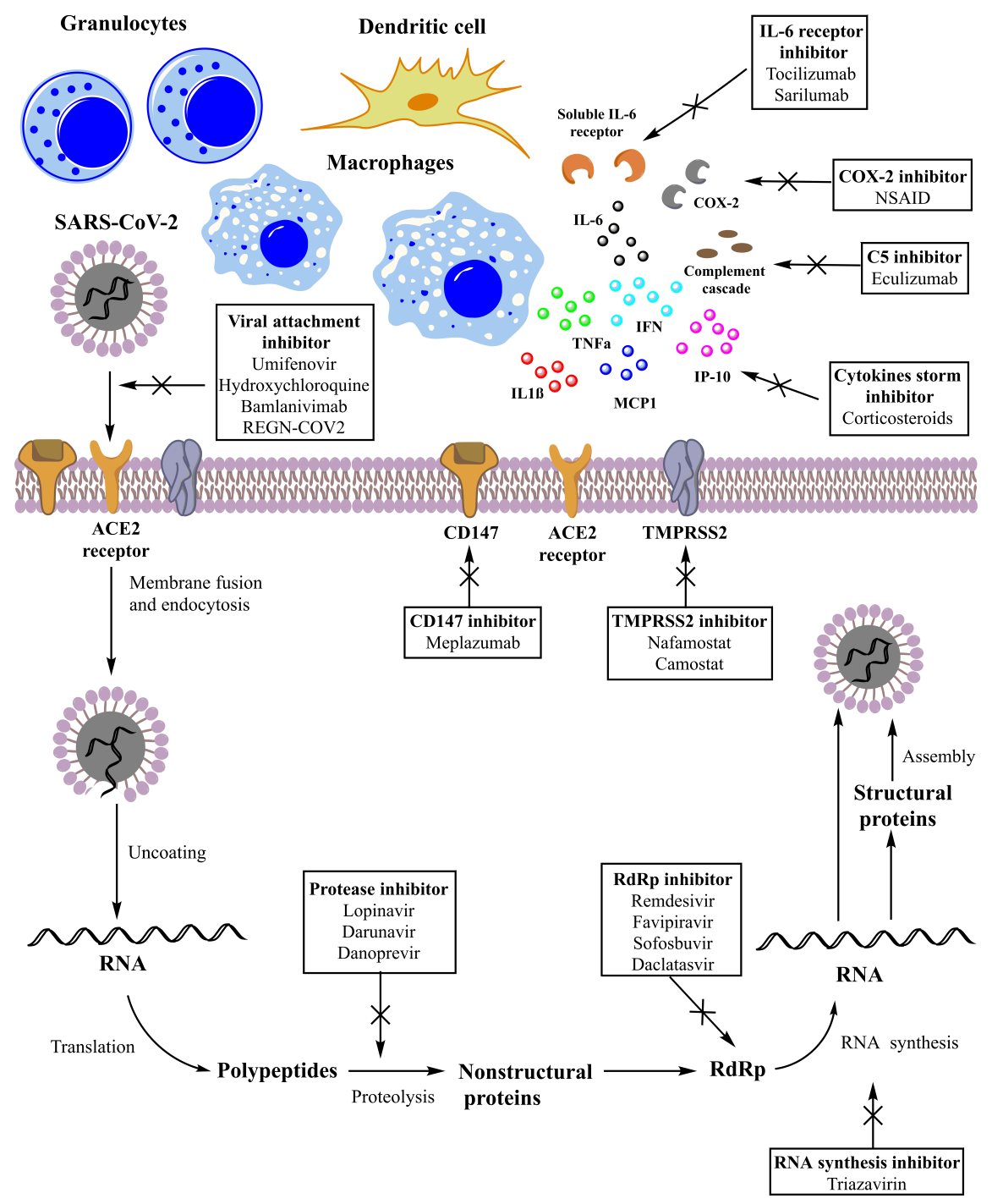

Figure 2. Main drugs evaluated for COVID-19

Table 1 . Vaccines in phase 3 of clinical research.

\begin{tabular}{llllll}
\hline $\begin{array}{l}\text { Manufacturer } \\
\text { of COVID-19 } \\
\text { vaccine }\end{array}$ & $\begin{array}{l}\text { Vaccine } \\
\text { platform }\end{array}$ & $\begin{array}{l}\text { Type of } \\
\text { candidate } \\
\text { vaccine }\end{array}$ & $\begin{array}{l}\text { Number of } \\
\text { doses and } \\
\text { timing of doses }\end{array}$ & $\begin{array}{l}\text { Estimated } \\
\text { Identifier }\end{array}$ & $\begin{array}{l}\text { Study } \\
\text { Completion } \\
\text { Date }\end{array}$ \\
\hline $\begin{array}{l}\text { University of } \\
\text { Oxford/AstraZenecaViral Vector }\end{array}$ & $\begin{array}{l}\text { Non-Replicating } \\
\text { ChAdOx1-S }\end{array}$ & $\begin{array}{l}2 \text { doses at day } 0 \\
\text { and } 28\end{array}$ & NCT04516746 & February 2023 \\
& & & ISRCTN89951424 & December \\
& & & & NCT04540393 March 2021 \\
& & & CTRI/2020/08/027 March 2021*
\end{tabular}




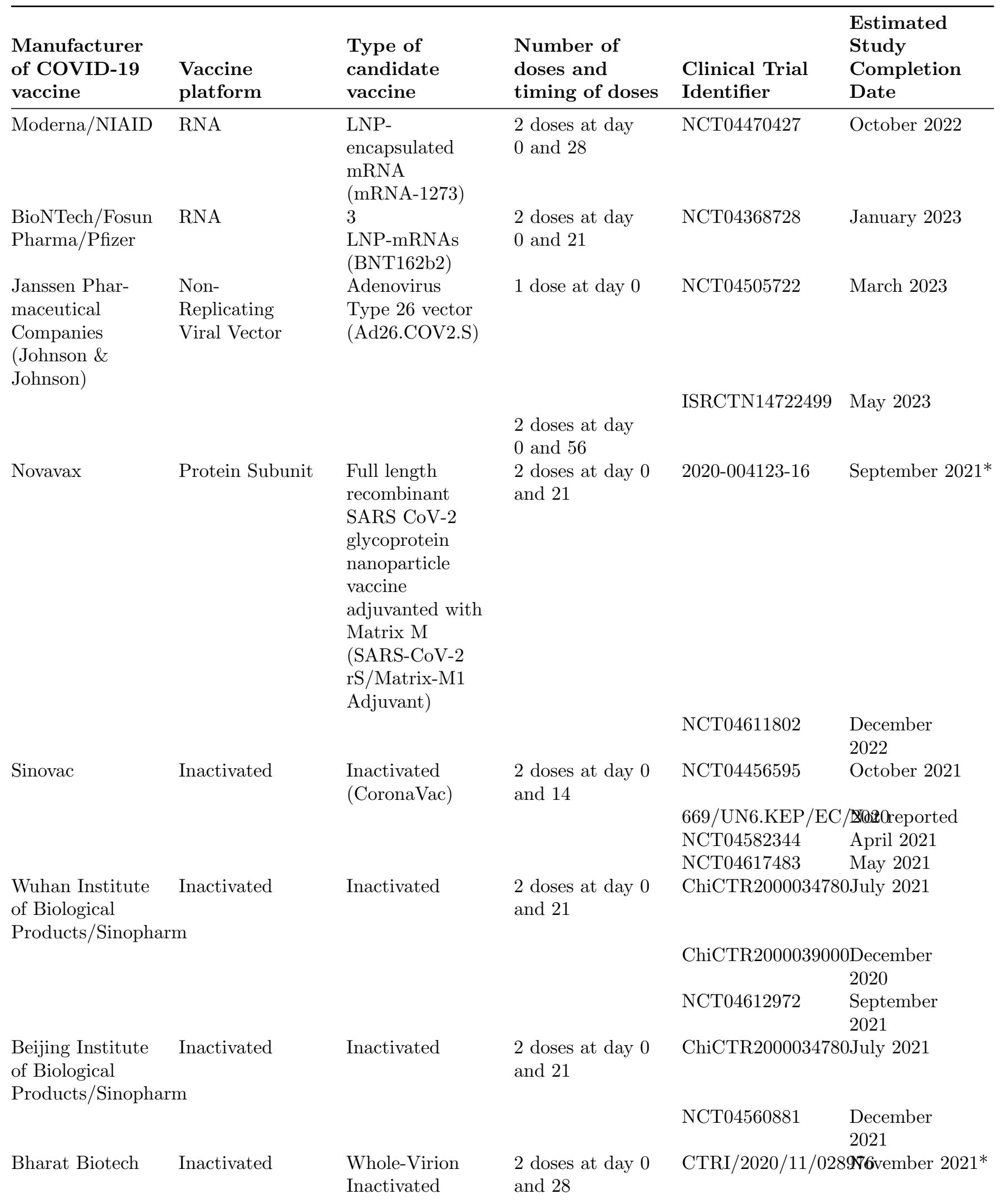




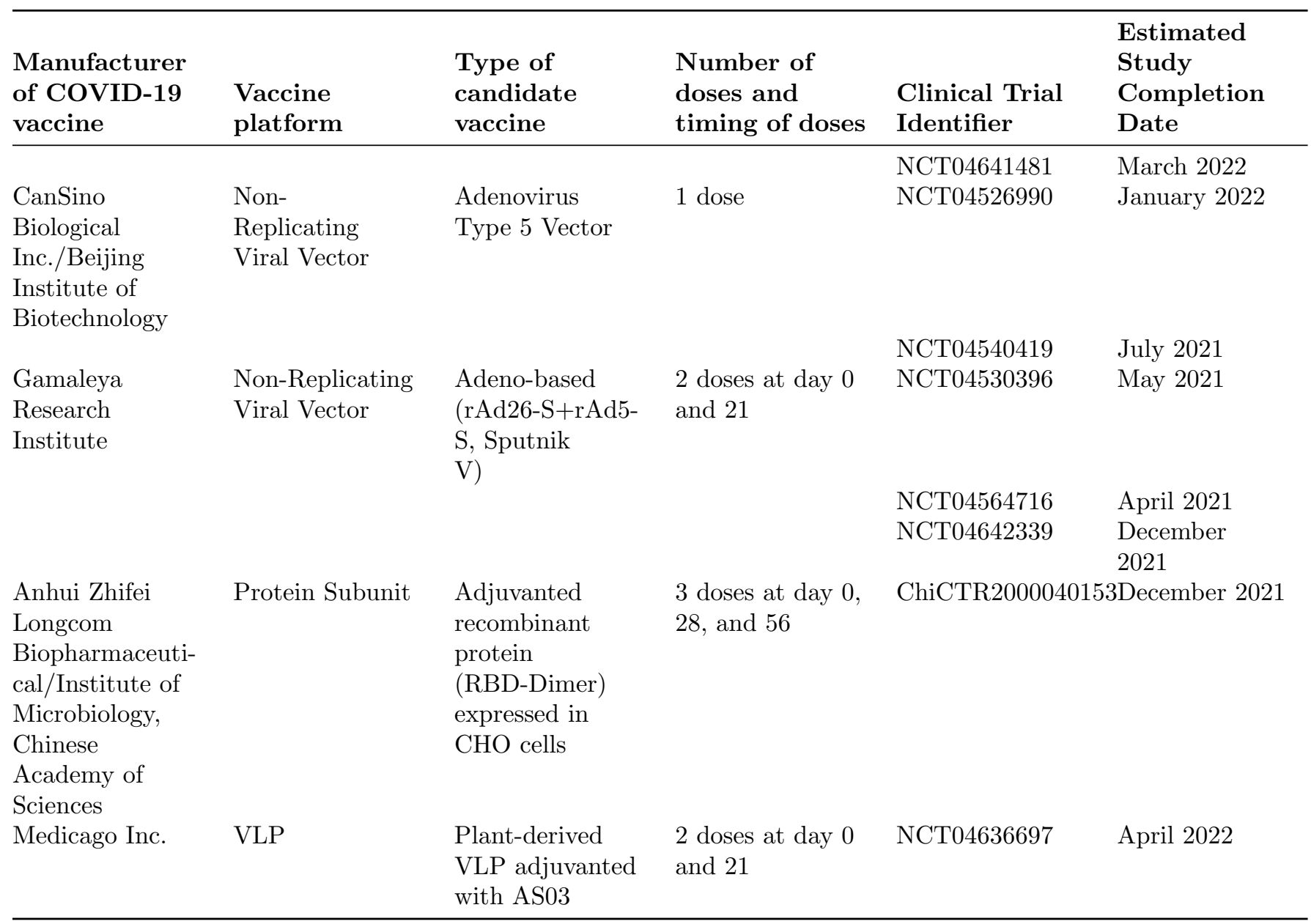

Lipid nanoparticle (LNP) Virus-Like Particles (VLP) *Dates computed based on the estimated trial duration.

\section{References}

Abbaspour Kasgari, H., Moradi, S., Shabani, A.M., Babamahmoodi, F., Davoudi Badabi, A.R., Davoudi, L., et al. (2020). Evaluation of the efficacy of sofosbuvir plus daclatasvir in combination with ribavirin for hospitalized COVID-19 patients with moderate disease compared with standard care: a single-centre, randomized controlled trial. J. Antimicrob. Chemother. 75 : 3373-3378.

Abu Esba, L.C., Alqahtani, R.A., Thomas, A., Shamas, N., Alswaidan, L., and Mardawi, G. (2020). Ibuprofen and NSAID Use in COVID-19 Infected Patients Is Not Associated with Worse Outcomes: A Prospective Cohort Study. Infect. Dis. Ther. 1-16.

Agostini, M.L., Andres, E.L., Sims, A.C., Graham, R.L., Sheahan, T.P., Lu, X., et al. (2018). Coronavirus susceptibility to the antiviral remdesivir (GS-5734) is mediated by the viral polymerase and the proofreading exoribonuclease. MBio 9 :.

Ali, N. (2020). Role of vitamin D in preventing of COVID-19 infection, progression and severity. J. Infect. Public Health 13 : 1373-1380.

Alshaeri, H.K., and Natto, Z.S. (2020). A contemporary look at COVID-19 medications: Available and potentially effective drugs. Eur. Rev. Med. Pharmacol. Sci. 24 : 9188-9195. 
American Association for Clinical Chemistry (2020). Disseminated Intravascular Coagulation in COVID19-Insights From the Front Lines | AACC.org.

American Society of Hematology (2020a). COVID-19 and Coagulopathy - Hematology.org.

American Society of Hematology (2020b). COVID-19 and VTE-Anticoagulation - Hematology.org.

Angus, D.C., Derde, L., Al-Beidh, F., Annane, D., Arabi, Y., Beane, A., et al. (2020). Effect of Hydrocortisone on Mortality and Organ Support in Patients with Severe COVID-19: The REMAP-CAP COVID-19 Corticosteroid Domain Randomized Clinical Trial. JAMA - J. Am. Med. Assoc. 324 : 1317-1329.

Annane, D., Heming, N., Grimaldi-Bensouda, L., Frémeaux-Bacchi, V., Vigan, M., Roux, A.L., et al. (2020). Eculizumab as an emergency treatment for adult patients with severe COVID-19 in the intensive care unit: A proof-of-concept study. EClinicalMedicine 28: 100590.

ANSM (2019). Anti-inflammatoires non stéroïdiens (AINS) et complications infectieuses graves - Point d'Information actualisé le 20/05/2020 - ANSM : Agence nationale de securite du medicament et des produits de sante.

Arabi, Y.M., Hajeer, A.H., Luke, T., Raviprakash, K., Balkhy, H., Johani, S., et al. (2016). Feasibility of using convalescent plasma immunotherapy for MERS-CoV infection, Saudi Arabia. Emerg. Infect. Dis.22 : $1554-1561$.

Asakura, H., and Ogawa, H. (2020). COVID-19-associated coagulopathy and disseminated intravascular coagulation. Int. J. Hematol. $1: 3$.

AstraZeneca (2020). AstraZeneca's COVID-19 vaccine authorised for emergency supply in the UK.

Atallah, B., Mallah, S.I., and AlMahmeed, W. (2020). Anticoagulation in COVID-19. Eur. Hear. J. Cardiovasc. Pharmacother. $6: 260-261$.

Ayerbe, L., Risco, C., and Ayis, S. (2020). The association between treatment with heparin and survival in patients with Covid-19. J. Thromb. Thrombolysis $50: 298-301$.

Baden, L.R., Sahly, H.M. El, Essink, B., Kotloff, K., Frey, S., Novak, R., et al. (2020). Efficacy and Safety of the mRNA-1273 SARS-CoV-2 Vaccine. N. Engl. J. Med. NEJMoa2035389.

Bairi, K. El, Trapani, D., Petrillo, A., Page, C. Le, Zbakh, H., Daniele, B., et al. (2020). Repurposing anticancer drugs for the management of COVID-19. Eur. J. Cancer 141: 40-61.

Bancos, S., Bernard, M.P., Topham, D.J., and Phipps, R.P. (2009). Ibuprofen and other widely used nonsteroidal anti-inflammatory drugs inhibit antibody production in human cells. Cell. Immunol. 258 : 18-28.

Beigel, J.H., Tomashek, K.M., Dodd, L.E., Mehta, A.K., Zingman, B.S., Kalil, A.C., et al. (2020). Remdesivir for the Treatment of Covid-19 - Final Report. N. Engl. J. Med.

Benucci, M., Giannasi, G., Cecchini, P., Gobbi, F.L., Damiani, A., Grossi, V., et al. (2020). COVID-19 pneumonia treated with Sarilumab: A clinical series of eight patients. J. Med. Virol. 92 : 2368-2370.

Bhattacharyya, A., Kumar, S., Sarma, P., Kaur, H., Prajapat, M., Shekhar, N., et al. (2020). Safety and efficacy of lopinavir/ ritonavir combination in COVID-19: A systematic review, meta-analysis, and meta-regression analysis. Indian J. Pharmacol. 52 : 313-323.

Bian, H., Zheng, Z.-H., Wei, D., Zhang, Z., Kang, W.-Z., Hao, C.-Q., et al. (2020). Meplazumab treats COVID-19 pneumonia: an open-labelled, concurrent controlled add-on clinical trial. MedRxiv 2020.03.21.20040691.

Bianconi, V., Violi, F., Fallarino, F., Pignatelli, P., Sahebkar, A., and Pirro, M. (2020). Is Acetylsalicylic Acid a Safe and Potentially Useful Choice for Adult Patients with COVID-19 ? Drugs 80 : 1383-1396. 
Bloch, E.M., Shoham, S., Casadevall, A., Sachais, B.S., Shaz, B., Winters, J.L., et al. (2020). Deployment of convalescent plasma for the prevention and treatment of COVID-19. J. Clin. Invest. $130: 2757-2765$.

Boulware, D.R., Pullen, M.F., Bangdiwala, A.S., Pastick, K.A., Lofgren, S.M., Okafor, E.C., et al. (2020). A Randomized Trial of Hydroxychloroquine as Postexposure Prophylaxis for Covid-19. N. Engl. J. Med. $383: 517-525$.

Boyle, A.J., Gangi, S. Di, Hamid, U.I., Mottram, L.J., McNamee, L., White, G., et al. (2015). Aspirin therapy in patients with acute respiratory distress syndrome (ARDS) is associated with reduced intensive care unit mortality: A prospective analysis. Crit. Care19 :.

Bruce, E., Barlow-Pay, F., Short, R., Vilches-Moraga, A., Price, A., McGovern, A., et al. (2020). Prior Routine Use of Non-Steroidal Anti-Inflammatory Drugs (NSAIDs) and Important Outcomes in Hospitalised Patients with COVID-19. J. Clin. Med. $9: 2586$.

Buijsers, B., Yanginlar, C., Maciej-Hulme, M.L., Mast, Q. de, and Vlag, J. van der (2020). Beneficial non-anticoagulant mechanisms underlying heparin treatment of COVID-19 patients. EBioMedicine 59 : 102969.

Cacciapuoti, S., Rosa, A. De, Gelzo, M., Megna, M., Raia, M., Pinchera, B., et al. (2020). Immunocytometric analysis of COVID patients: A contribution to personalized therapy? Life Sci. 261 : 118355.

Cai, Q., Yang, M., Liu, D., Chen, J., Shu, D., Xia, J., et al. (2020). Experimental Treatment with Favipiravir for COVID-19: An Open-Label Control Study. Engineering.

Caly, L., Druce, J.D., Catton, M.G., Jans, D.A., and Wagstaff, K.M. (2020). The FDA-approved drug ivermectin inhibits the replication of SARS-CoV-2 in vitro. Antiviral Res. 178 :.

Camprubi, D., Almuedo-Riera, A., Marti-Soler, H., Soriano, A., Hurtado, J.C., Subira, C., et al. (2020). Lack of efficacy of standard doses of ivermectin in severe COVID-19 patients. PLoS One 15 : e0242184.

Cantini, F., Niccoli, L., Matarrese, D., Nicastri, E., Stobbione, P., and Goletti, D. (2020a). Baricitinib therapy in COVID-19: A pilot study on safety and clinical impact. J. Infect. 81 : 318-356.

Cantini, F., Niccoli, L., Nannini, C., Matarrese, D., Natale, M.E. Di, Lotti, P., et al. (2020b). Beneficial impact of Baricitinib in COVID-19 moderate pneumonia; multicentre study. J. Infect. 81 : 647-679.

Cao, B., Wang, Y., Wen, D., Liu, W., Wang, J., Fan, G., et al. (2020). A Trial of Lopinavir-Ritonavir in Adults Hospitalized with Severe Covid-19. N. Engl. J. Med. 382 : 1787-1799.

Capoluongo, E.D., Amato, F., and Castaldo, G. (2020). The friendly use of chloroquine in the COVID-19 disease: A warning for the G6PD-deficient males and for the unaware carriers of pathogenic alterations of the G6PD gene. Clin. Chem. Lab. Med. 58 : 1162-1164.

Capuano, A., Rossi, F., and Paolisso, G. (2020a). Covid-19 Kills More Men Than Women: An Overview of Possible Reasons. Front. Cardiovasc. Med.7 : 131.

Capuano, A., Scavone, C., Racagni, G., and Scaglione, F. (2020b). NSAIDs in patients with viral infections, including Covid-19: Victims or perpetrators? Pharmacol. Res. 157 : 104849.

Chen, C., Zhang, Y., Huang, J., Yin, P., Cheng, Z., Wu, J., et al. (2020a). Favipiravir versus Arbidol for COVID-19: A Randomized Clinical Trial.

CHEN, J., LIU, D., LIU, L., LIU, P., XU, Q., XIA, L., et al. (2020). A pilot study of hydroxychloroquine in treatment of patients with common coronavirus disease-19 (COVID-19). J Zhejiang Univ (Med Sci) 49 : $1-10$.

Chen, J., Xia, L., Liu, L., Xu, Q., Ling, Y., Huang, D., et al. (2020b). Antiviral activity and safety of darunavir/Cobicistat for the treatment of COVID-19. Open Forum Infect. Dis. 7 :. 
Chen, P., Nirula, A., Heller, B., Gottlieb, R.L., Boscia, J., Morris, J., et al. (2020c). SARS-CoV-2 Neutralizing Antibody LY-CoV555 in Outpatients with Covid-19. N. Engl. J. Med.

Chen, W., Janz, D.R., Bastarache, J.A., May, A.K., O'Neal, H.R., Bernard, G.R., et al. (2015). Prehospital aspirin use is associated with reduced risk of acute respiratory distress syndrome in critically ill patients: A propensity-adjusted analysis. Crit. Care Med. 43 : 801-807.

Cheng, Y., Wong, R., Soo, Y.O.Y., Wong, W.S., Lee, C.K., Ng, M.H.L., et al. (2005). Use of convalescent plasma therapy in SARS patients in Hong Kong. Eur. J. Clin. Microbiol. Infect. Dis. 24 : 44-46.

Choi, M.J., Kang, M., Shin, S.Y., Noh, J.Y., Cheong, H.J., Kim, W.J., et al. (2020). Comparison of antiviral effect for mild-to-moderate COVID-19 cases between lopinavir/ritonavir versus hydroxychloroquine: A nationwide propensity score-matched cohort study. Int. J. Infect. Dis.

Chow, J.H., Khanna, A.K., Kethireddy, S., Yamane, D., Levine, A., Jackson, A.M., et al. (2020). Aspirin Use is Associated with Decreased Mechanical Ventilation, ICU Admission, and In-Hospital Mortality in Hospitalized Patients with COVID-19. Anesth. Analg. Publish Ah :

Cingolani, A., Tummolo, A.M., Montemurro, G., Gremese, E., Larosa, L., Cipriani, M.C., et al. (2020). Baricitinib as rescue therapy in a patient with COVID-19 with no complete response to sarilumab. Infection 48 : 767-771.

Cordon-Cardo, C., Pujadas, E., Wajnberg, A., Sebra, R., Patel, G., Firpo-Betancourt, A., et al. (2020). COVID-19: Staging of a New Disease. Cancer Cell 38 : 594-597.

D'avolio, A., Avataneo, V., Manca, A., Cusato, J., Nicolo, A. De, Lucchini, R., et al. (2020). 25hydroxyvitamin D concentrations are lower in patients with positive PCR for SARS-CoV-2. Nutrients12 $\therefore$

Deeks, E.D. (2018). Darunavir/Cobicistat/Emtricitabine/Tenofovir Alafenamide: A Review in HIV-1 Infection. Drugs 78 : 1013-1024.

Deng, L., Li, C., Zeng, Q., Liu, X., Li, X., Zhang, H., et al. (2020). Arbidol combined with LPV/r versus LPV/r alone against Corona Virus Disease 2019: A retrospective cohort study. J. Infect. 81 : e1-e5.

Dequin, P.F., Heming, N., Meziani, F., Plantefeve, G., Voiriot, G., Badie, J., et al. (2020). Effect of Hydrocortisone on 21-Day Mortality or Respiratory Support among Critically Ill Patients with COVID-19: A Randomized Clinical Trial. JAMA - J. Am. Med. Assoc. 324 : 1298-1306.

Doi, K., Ikeda, M., Hayase, N., Moriya, K., Morimura, N., Maehara, H., et al. (2020). Nafamostat mesylate treatment in combination with favipiravir for patients critically ill with Covid-19: a case series. Crit. Care $24:$.

Duan, K., Liu, B., Li, C., Zhang, H., Yu, T., Qu, J., et al. (2020). Effectiveness of convalescent plasma therapy in severe COVID-19 patients. Proc. Natl. Acad. Sci. U. S. A. 117 : 9490-9496.

Eli Lilly and Company Lilly's neutralizing antibody bamlanivimab (LY-CoV555) receives interim authorization from Health Canada as a treatment for COVID-19 | Eli Lilly and Company.

Elsawah, H.K., Elsokary, M.A., Abdallah, M.S., and ElShafie, A.H. (2020). Efficacy and safety of remdesivir in hospitalized Covid-19 patients: Systematic review and meta-analysis including network meta-analysis. Rev. Med. Virol.

Erlich, J.M., Talmor, D.S., Cartin-Ceba, R., Gajic, O., and Kor, D.J. (2011). Prehospitalization antiplatelet therapy is associated with a reduced incidence of acute lung injury: A population-based cohort study. Chest $139: 289-295$.

Eslami, G., Mousaviasl, S., Radmanesh, E., Jelvay, S., Bitaraf, S., Simmons, B., et al. (2020). The impact of sofosbuvir/daclatasvir or ribavirin in patients with severe COVID-19. J. Antimicrob. Chemother.75 : 
$3366-3372$.

European Medicine agency (2020). COVID-19: chloroquine and hydroxychloroquine only to be used in clinical trials or emergency use programmes | European Medicines Agency.

European Medicine Agency Update on remdesivir - EMA will evaluate new data from Solidarity trial | European Medicines Agency.

European Medicine Agency (2020a). COVID-19: reminder of risk of serious side effects with chloroquine and hydroxychloroquine.

European Medicine Agency (2020b). COVID-19: reminder of the risks of chloroquine and hydroxychloroquine | European Medicines Agency.

European Medicine Agency (2020c). EMA endorses use of dexamethasone in COVID-19 patients on oxygen or mechanical ventilation | European Medicines Agency.

European Medicine Agency (2020d). Meeting highlights from the Pharmacovigilance Risk Assessment Committee (PRAC) 23-26 November 2020 | European Medicines Agency.

Favalli, E.G., Biggioggero, M., Maioli, G., and Caporali, R. (2020). Baricitinib for COVID-19: a suitable treatment? Lancet Infect. Dis.20 : 1012-1013.

Fehr, A.R., and Perlman, S. (2015). Coronaviruses: An overview of their replication and pathogenesis. In Coronaviruses: Methods and Protocols, (Springer New York), pp 1-23.

Ferrara, N., Hillan, K.J., and Novotny, W. (2005). Bevacizumab (Avastin), a humanized anti-VEGF monoclonal antibody for cancer therapy. Biochem. Biophys. Res. Commun. 333 : 328-335.

Food and Drug Administration Coronavirus (COVID-19) Update: FDA Authorizes Monoclonal Antibodies for Treatment of COVID-19 | FDA.

Gao, G., Wang, A., Wang, S., Qian, F., Chen, M., Yu, F., et al. (2020). Brief Report: Retrospective Evaluation on the Efficacy of Lopinavir/Ritonavir and Chloroquine to Treat Nonsevere COVID-19 Patients. J. Acquir. Immune Defic. Syndr. 85 : 239-243.

Gautret, P., Lagier, J.C., Parola, P., Hoang, V.T., Meddeb, L., Mailhe, M., et al. (2020). Hydroxychloroquine and azithromycin as a treatment of COVID-19: results of an open-label non-randomized clinical trial. Int. J. Antimicrob. Agents $56: 105949$.

Goldman, J.D., Lye, D.C.B., Hui, D.S., Marks, K.M., Bruno, R., Montejano, R., et al. (2020). Remdesivir for 5 or 10 Days in Patients with Severe Covid-19. N. Engl. J. Med.

Gozzo, L., Viale, P., Longo, L., Vitale, D.C., and Drago, F. (2020). The Potential Role of Heparin in Patients With COVID-19: Beyond the Anticoagulant Effect. A Review. Front. Pharmacol. 11 :.

Gralinski, L.E., Sheahan, T.P., Morrison, T.E., Menachery, V.D., Jensen, K., Leist, S.R., et al. (2018). Complement activation contributes to severe acute respiratory syndrome coronavirus pathogenesis. MBio 9 $\therefore$

Grein, J., Ohmagari, N., Shin, D., Diaz, G., Asperges, E., Castagna, A., et al. (2020). Compassionate Use of Remdesivir for Patients with Severe Covid-19. N. Engl. J. Med. 382 : 2327-2336.

Gremese, E., Cingolani, A., Bosello, S.L., Alivernini, S., Tolusso, B., Perniola, S., et al. (2020). Sarilumab use in severe SARS-CoV-2 pneumonia. EClinicalMedicine $27:$

Guo, Y.R., Cao, Q.D., Hong, Z.S., Tan, Y.Y., Chen, S.D., Jin, H.J., et al. (2020a). The origin, transmission and clinical therapies on coronavirus disease 2019 (COVID-19) outbreak- A n update on the status. Mil. Med. Res. 7 :. 
Guo, Y.R., Cao, Q.D., Hong, Z.S., Tan, Y.Y., Chen, S.D., Jin, H.J., et al. (2020b). The origin, transmission and clinical therapies on coronavirus disease 2019 (COVID-19) outbreak - an update on the status. Mil. Med. Res. 7: 11 .

Hastie, C.E., Mackay, D.F., Ho, F., Celis-Morales, C.A., Katikireddi, S.V., Niedzwiedz, C.L., et al. (2020). Vitamin D concentrations and COVID-19 infection in UK Biobank. Diabetes Metab. Syndr. Clin. Res. Rev.14 : 561-565.

Hoffmann, M., Kleine-Weber, H., Schroeder, S., Kruger, N., Herrler, T., Erichsen, S., et al. (2020). SARSCoV-2 Cell Entry Depends on ACE2 and TMPRSS2 and Is Blocked by a Clinically Proven Protease Inhibitor. Cell181 : 271-280.e8.

Horby, P., Lim, W.S., Emberson, J.R., Mafham, M., Bell, J.L., Linsell, L., et al. (2020a). Dexamethasone in Hospitalized Patients with Covid-19 — Preliminary Report. N. Engl. J. Med.

Horby, P.W., Mafham, M., Bell, J.L., Linsell, L., Staplin, N., Emberson, J., et al. (2020b). Lopinavirritonavir in patients admitted to hospital with COVID-19 (RECOVERY): a randomised, controlled, openlabel, platform trial. Lancet $396: 1345-1352$.

Huang, M., Tang, T., Pang, P., Li, M., Ma, R., Lu, J., et al. (2020). Treating COVID-19 with Chloroquine. J. Mol. Cell Biol. 12 : 322-325.

Hung, I.F.N., Lung, K.C., Tso, E.Y.K., Liu, R., Chung, T.W.H., Chu, M.Y., et al. (2020). Triple combination of interferon beta-1b, lopinavir-ritonavir, and ribavirin in the treatment of patients admitted to hospital with COVID-19: an open-label, randomised, phase 2 trial. Lancet 395 : 1695-1704.

Italian Medicine Agency RIASSUNTO DELLE CARATTERISTICHE DEL PRODOTTO.

Italian Medicine Agency (2020a). COVID-19: STUDIO RANDOMIZZATO ITALIANO, NESSUN BENEFICIO DAL TOCILIZUMAB.

Italian Medicine Agency (2020b). Idrossiclorochina nella terapia dei pazienti adulti con COVID-19 .

Ivashchenko, A.A., Dmitriev, K.A., Vostokova, N. V, Azarova, V.N., Blinow, A.A., Egorova, A.N., et al. (2020). AVIFAVIR for Treatment of Patients with Moderate COVID-19: Interim Results of a Phase II/III Multicenter Randomized Clinical Trial. Clin. Infect. Dis.

Iwasaka, S., Shono, Y., Tokuda, K., Nakashima, K., Yamamoto, Y., Maki, J., et al. (2020). Clinical improvement in a patient with severe coronavirus disease 2019 after administration of hydroxychloroquine and continuous hemodiafiltlation with nafamostat mesylate. J. Infect. Chemother. 26 : 1319-1323.

Janssen (2020). Janssen begins second Phase III Covid-19 vaccine trial .

Jeronimo, C.M.P., Farias, M.E.L., Val, F.F.A., Sampaio, V.S., Alexandre, M.A.A., Melo, G.C., et al. (2020). Methylprednisolone as Adjunctive Therapy for Patients Hospitalized With Coronavirus Disease 2019 (COVID-19; Metcovid): A Randomized, Double-blind, Phase IIb, Placebo-controlled Trial. Clin. Infect. Dis.

Jovic, T.H., Ali, S.R., Ibrahim, N., Jessop, Z.M., Tarassoli, S.P., Dobbs, T.D., et al. (2020). Could vitamins help in the fight against covid-19? Nutrients 12 : 1-30.

Karolyi, M., Pawelka, E., Mader, T., Omid, S., Kelani, H., Ely, S., et al. (2020). Hydroxychloroquine versus lopinavir/ritonavir in severe COVID-19 patients: Results from a real-life patient cohort. Wien. Klin. Wochenschr.

Khamis, F., Naabi, H. Al, Lawati, A. Al, Ambusaidi, Z., Sharji, M. Al, Barwani, U. Al, et al. (2020). Randomized Controlled Open Label Trial on the Use of Favipiravir Combined with Inhaled Interferon beta1b in Hospitalized Patients with Moderate to Severe COVID-19 Pneumonia. Int. J. Infect. Dis. 
Kim, E.J., Choi, S.H., Park, J.S., Kwon, Y.S., Lee, J., Kim, Y., et al. (2020). Use of darunavir-cobicistat as a treatment option for critically ill patients with sars-cov-2 infection. Yonsei Med. J. 61 : 826-830.

Klok, F.A., Kruip, M.J.H.A., Meer, N.J.M. van der, Arbous, M.S., Gommers, D.A.M.P.J., Kant, K.M., et al. (2020). Incidence of thrombotic complications in critically ill ICU patients with COVID-19. Thromb. Res.191: 145-147.

Kor, D.J., Carter, R.E., Park, P.K., Festic, E., Banner-Goodspeed, V.M., Hinds, R., et al. (2016). Effect of aspirin on development of ARDS in at-risk patients presenting to the emergency department the LIPS-a randomized clinical trial. JAMA - J. Am. Med. Assoc. 315 : 2406-2414.

Kor, D.J., Erlich, J., Gong, M.N., Malinchoc, M., Carter, R.E., Gajic, O., et al. (2011). Association of prehospitalization aspirin therapy and acute lung injury: Results of a multicenter international observational study of at-risk patients. Crit. Care Med. 39 : 2393-2400.

Kraft, C.S., Hewlett, A.L., Koepsell, S., Winkler, A.M., Kratochvil, C.J., Larson, L., et al. (2015). The Use of TKM-100802 and Convalescent Plasma in 2 Patients With Ebola Virus Disease in the United States. Clin. Infect. Dis. $61:$ 496-502.

Laurence, J., Mulvey, J.J., Seshadri, M., Racanelli, A., Harp, J., Schenck, E.J., et al. (2020). Anticomplement C5 therapy with eculizumab in three cases of critical COVID-19. Clin. Immunol. 219 :.

Lecronier, M., Beurton, A., Burrel, S., Haudebourg, L., Deleris, R., Marec, J. Le, et al. (2020). Comparison of hydroxychloroquine, lopinavir/ritonavir, and standard of care in critically ill patients with SARS-CoV-2 pneumonia: An opportunistic retrospective analysis. Crit. Care 24 :.

Leppkes, M., Knopf, J., Naschberger, E., Lindemann, A., Singh, J., Herrmann, I., et al. (2020). Vascular occlusion by neutrophil extracellular traps in COVID-19. EBioMedicine 58 :.

Leuchte, H.H., Baezner, C., Baumgartner, R.A., Bevec, D., Bacher, G., Neurohr, C., et al. (2008). Inhalation of vasoactive intestinal peptide in pulmonary hypertension. Eur. Respir. J. 32 : 1289-1294.

Levi, M., Thachil, J., Iba, T., and Levy, J.H. (2020). Coagulation abnormalities and thrombosis in patients with COVID-19. Lancet Haematol.7 : e438-e440.

Lewis, T.C., Adhikari, S., Tatapudi, V., Holub, M., Kunichoff, D., Troxel, A.B., et al. (2020). A PropensityMatched Cohort Study of Tocilizumab in Patients With Coronavirus Disease 2019. Crit. Care Explor. 2 : $\mathrm{e} 0283$.

Li, H., Liu, L., Zhang, D., Xu, J., Dai, H., Tang, N., et al. (2020a). SARS-CoV-2 and viral sepsis: observations and hypotheses. Lancet395 : 1517-1520.

Li, L., Zhang, W., Hu, Y., Tong, X., Zheng, S., Yang, J., et al. (2020b). Effect of Convalescent Plasma Therapy on Time to Clinical Improvement in Patients with Severe and Life-threatening COVID-19: A Randomized Clinical Trial. JAMA - J. Am. Med. Assoc. 324 : 460-470.

Li, T., Lu, H., and Zhang, W. (2020c). Clinical observation and management of COVID-19 patients. Emerg. Microbes Infect. $9: 687-690$.

Li, X., and Ma, X. (2017). The role of heparin in sepsis: much more than just an anticoagulant. Br. J. Haematol. 179 : 389-398.

Li, Y., Xie, Z., Lin, W., Cai, W., Wen, C., Guan, Y., et al. (2020d). Efficacy and Safety of Lopinavir/Ritonavir or Arbidol in Adult Patients with Mild/Moderate COVID-19: An Exploratory Randomized Controlled Trial. Med 0 :

Liu, C., Zhou, Q., Li, Y., Garner, L. V., Watkins, S.P., Carter, L.J., et al. (2020a). Research and Development on Therapeutic Agents and Vaccines for COVID-19 and Related Human Coronavirus Diseases. ACS Cent. Sci. 6 : 315-331. 
Liu, S.T.H., Lin, H.M., Baine, I., Wajnberg, A., Gumprecht, J.P., Rahman, F., et al. (2020b). Convalescent plasma treatment of severe COVID-19: a propensity score-matched control study. Nat. Med.26 : 1708-1713.

Lodigiani, C., Iapichino, G., Carenzo, L., Cecconi, M., Ferrazzi, P., Sebastian, T., et al. (2020). Venous and arterial thromboembolic complications in COVID-19 patients admitted to an academic hospital in Milan, Italy. Thromb. Res. 191: 9-14.

Lother, S.A., Abassi, M., Agostinis, A., Bangdiwala, A.S., Cheng, M.P., Drobot, G., et al. (2020). Postexposure prophylaxis or pre-emptive therapy for severe acute respiratory syndrome coronavirus 2 (SARSCoV-2): study protocol for a pragmatic randomized-controlled trial. Can. J. Anesth. 67 : 1201-1211.

Lou, Y., Liu, L., Yao, H., Hu, X., Su, J., Xu, K., et al. (2020). Clinical Outcomes and Plasma Concentrations of Baloxavir Marboxil and Favipiravir in COVID-19 Patients: An Exploratory Randomized, Controlled Trial. Eur. J. Pharm. Sci. 105631.

Marietta, M., Ageno, W., Artoni, A., Candia, E. De, Gresele, P., Marchetti, M., et al. (2020). COVID-19 and haemostasis: A position paper from Italian Society on Thrombosis and Haemostasis (SISET). Blood Transfus. $18: 167-169$.

Mascolo, A., Berrino, P.M., Gareri, P., Castagna, A., Capuano, A., Manzo, C., et al. (2018). Neuropsychiatric clinical manifestations in elderly patients treated with hydroxychloroquine: a review article. Inflammopharmacology $26: 1141-1149$.

Mascolo, A., Scavone, C., Rafaniello, C., Ferrajolo, C., Racagni, G., Berrino, L., et al. (2020). Reninangiotensin system and coronavirus disease 2019: A narrative review. Front. Cardiovasc. Med. 7 :.

McGonagle, D., O’Donnell, J.S., Sharif, K., Emery, P., and Bridgewood, C. (2020). Immune mechanisms of pulmonary intravascular coagulopathy in COVID-19 pneumonia. Lancet Rheumatol. 2 : e437-e445.

Meyer, S. De, Bojkova, D., Cinatl, J., Damme, E. Van, Buyck, C., Loock, M. Van, et al. (2020). Lack of antiviral activity of darunavir against SARS-CoV-2. Int. J. Infect. Dis. 97: 7-10.

Ministere de la Sante (2020). Liste des messages d'alertes.

Mitchell, F. (2020). Vitamin-D and COVID-19: do deficient risk a poorer outcome? Lancet Diabetes Endocrinol. $8: 570$.

Moderna (2020). Moderna Announces Primary Efficacy Analysis in Phase 3 COVE Study for Its COVID-19 Vaccine Candidate and Filing Today with U.S. FDA for Emergency Use Authorization | Moderna, Inc.

Molina, J.M., Delaugerre, C., Goff, J. Le, Mela-Lima, B., Ponscarme, D., Goldwirt, L., et al. (2020). No evidence of rapid antiviral clearance or clinical benefit with the combination of hydroxychloroquine and azithromycin in patients with severe COVID-19 infection. Med. Mal. Infect. $50: 384$.

monde, Le (2020). Le ministre de la sante deconseille l'ibuprofene contre le coronavirus.

Montesarchio, V., Parella, R., Iommelli, C., Bianco, A., Manzillo, E., Fraganza, F., et al. (2020). Outcomes and biomarker analyses among patients with COVID-19 treated with interleukin 6 (IL-6) receptor antagonist sarilumab at a single institution in Italy. J. Immunother. Cancer $8: 1089$.

Moores, L.K., Tritschler, T., Brosnahan, S., Carrier, M., Collen, J.F., Doerschug, K., et al. (2020). Prevention, Diagnosis, and Treatment of VTE in Patients With Coronavirus Disease 2019: CHEST Guideline and Expert Panel Report. Chest 158 : 1143-1163.

Nadkarni, G.N., Lala, A., Bagiella, E., Chang, H.L., Moreno, P.R., Pujadas, E., et al. (2020). Anticoagulation, Bleeding, Mortality, and Pathology in Hospitalized Patients With COVID-19. J. Am. Coll. Cardiol.76 : 1815-1826. 
Nicolini, L.A., Mikulska, M., Signori, A., Biagio, A. Di, Portunato, F., Vena, A., et al. (2020). Reply to: "Antiviral activity and safety of darunavir/cobicistat for treatment of COVID-19". Open Forum Infect. Dis. $7:$.

NIH (2020). Antithrombotic Therapy | COVID-19 Treatment Guidelines.

Niriella, M.A., Ediriweera, D.S., Silva, A.P. De, Premarathne, R., Balasooriya, P., Duminda, K.D., et al. (2020). Hydroxychloroquine for post-exposure prophylaxis of COVID-19 among naval personnel in Sri Lanka: Study protocol for a randomized, controlled trial. Trials21 : 748 .

Paranjpe, I., Fuster, V., Lala, A., Russak, A.J., Glicksberg, B.S., Levin, M.A., et al. (2020). Association of Treatment Dose Anticoagulation With In-Hospital Survival Among Hospitalized Patients With COVID-19. J. Am. Coll. Cardiol. $76: 122-124$.

Pasquini, Z., Montalti, R., Temperoni, C., Canovari, B., Mancini, M., Tempesta, M., et al. (2020). Effectiveness of remdesivir in patients with COVID-19 under mechanical ventilation in an Italian ICU. J. Antimicrob. Chemother. 75 : 3359-3365.

Pavoni, V., Gianesello, L., Pazzi, M., Stera, C., Meconi, T., and Frigieri, F.C. (2020). Venous thromboembolism and bleeding in critically ill COVID-19 patients treated with higher than standard low molecular weight heparin doses and aspirin: A call to action. Thromb. Res.196 : 313-317.

Perrone, F., Piccirillo, M.C., Ascierto, P.A., Salvarani, C., Parrella, R., Marata, A.M., et al. (2020). Tocilizumab for patients with COVID-19 pneumonia. The single-arm TOCIVID-19 prospective trial. J. Transl. Med.18 :.

Pizzini, A., Aichner, M., Sahanic, S., Bohm, A., Egger, A., Hoermann, G., et al. (2020). Impact of vitamin d deficiency on covid-19 - a prospective analysis from the covild registry. Nutrients 12 : 1-9.

Polack, F.P., Thomas, S.J., Kitchin, N., Absalon, J., Gurtman, A., Lockhart, S., et al. (2020). Safety and Efficacy of the BNT162b2 mRNA Covid-19 Vaccine. N. Engl. J. Med.

Qian, Z., Travanty, E.A., Oko, L., Edeen, K., Berglund, A., Wang, J., et al. (2013). Innate immune response of human alveolar type II cells infected with severe acute respiratory syndrome-coronavirus. Am. J. Respir. Cell Mol. Biol. 48 : 742-748.

Rajter, J.C., Sherman, M.S., Fatteh, N., Vogel, F., Sacks, J., and Rajter, J.-J. (2020). Use of Ivermectin Is Associated With Lower Mortality in Hospitalized Patients With Coronavirus Disease 2019. Chest.

Rattanaumpawan, P., Jirajariyavej, S., Lerdlamyong, K., Palavutitotai, N., and Saiyarin, J. (2020). Realworld Experience with Favipiravir for Treatment of COVID-19 in Thailand: Results from a Multi-center Observational Study. MedRxiv 2020.06.24.20133249.

Regeneron Pharmaceuticals, I. Regeneron's REGN-COV2 Antibody Cocktail Reduced Viral Levels and Improved Symptoms in Non-Hospitalized COVID-19 Patients | Regeneron Pharmaceuticals Inc.

Rinott, E., Kozer, E., Shapira, Y., Bar-Haim, A., and Youngster, I. (2020). Ibuprofen use and clinical outcomes in COVID-19 patients. Clin. Microbiol. Infect. 26 : 1259.e5-1259.e7.

Roche (2020). Roche - Roche provides an update on the phase III COVACTA trial of Actemra/RoActemra in hospitalised patients with severe COVID-19 associated pneumonia.

Rojas, M., Rodriguez, Y., Monsalve, D.M., Acosta-Ampudia, Y., Camacho, B., Gallo, J.E., et al. (2020). Convalescent plasma in Covid-19: Possible mechanisms of action. Autoimmun. Rev. 19 :.

Sadeghi, A., Ali Asgari, A., Norouzi, A., Kheiri, Z., Anushirvani, A., Montazeri, M., et al. (2020). Sofosbuvir and daclatasvir compared with standard of care in the treatment of patients admitted to hospital with moderate or severe coronavirus infection (COVID-19): a randomized controlled trial. J. Antimicrob. Chemother. $75: 3379-3385$. 
Sanofi (2020a). Sanofi and GSK announce a delay in their adjuvanted recombinant protein-based COVID-19 vaccine program to improve immune response in the elderly - Sanofi.

Sanofi (2020b). Sanofi provides update on Kevzara(r) (sarilumab) Phase 3 trial in severe and critically ill COVID-19 patients outside the U.S. - Sanofi.

Scavone, C., Brusco, S., Bertini, M., Sportiello, L., Rafaniello, C., Zoccoli, A., et al. (2020). Current pharmacological treatments for COVID-19: What's next? Br. J. Pharmacol. 177 : 4813-4824.

Scialo, F., Daniele, A., Amato, F., Pastore, L., Matera, M.G., Cazzola, M., et al. (2020). ACE2: The Major Cell Entry Receptor for SARS-CoV-2. Lung 198 : 867-877.

Scott, L.J. (2017). Tocilizumab: A Review in Rheumatoid Arthritis. Drugs77 : 1865-1879.

Sheahan, T.P., Sims, A.C., Leist, S.R., Schafer, A., Won, J., Brown, A.J., et al. (2020). Comparative therapeutic efficacy of remdesivir and combination lopinavir, ritonavir, and interferon beta against MERSCoV. Nat. Commun. 11 :

Shen, C., Wang, Z., Zhao, F., Yang, Y., Li, J., Yuan, J., et al. (2020). Treatment of 5 Critically Ill Patients with COVID-19 with Convalescent Plasma. JAMA - J. Am. Med. Assoc. 323 : 1582-1589.

SHI, C., WANG, C., WANG, H., YANG, C., CAI, F., ZENG, F., et al. (2020). The potential of low molecular weight heparin to mitigate cytokine storm in severe COVID-19 patients: a retrospective clinical study. Rappaport Fac. Med. 2020.03.28.20046144.

Shrestha, D.B., Budhathoki, P., Khadka, S., Shah, P.B., Pokharel, N., and Rashmi, P. (2020). Favipiravir versus other antiviral or standard of care for COVID-19 treatment: a rapid systematic review and metaanalysis. Virol. J. 17: 141.

Simonovich, V.A., Burgos Pratx, L.D., Scibona, P., Beruto, M. V., Vallone, M.G., Vazquez, C., et al. (2020). A Randomized Trial of Convalescent Plasma in Covid-19 Severe Pneumonia. N. Engl. J. Med.

Skipper, C.P., Pastick, K.A., Engen, N.W., Bangdiwala, A.S., Abassi, M., Lofgren, S.M., et al. (2020). Hydroxychloroquine in Nonhospitalized Adults With Early COVID-19. Ann. Intern. Med. 173 : 623-631.

Spinner, C.D., Gottlieb, R.L., Criner, G.J., Arribas Lopez, J.R., Cattelan, A.M., Soriano Viladomiu, A., et al. (2020). Effect of Remdesivir vs Standard Care on Clinical Status at 11 Days in Patients with Moderate COVID-19: A Randomized Clinical Trial. JAMA - J. Am. Med. Assoc. 324 : 1048-1057.

Sterne, J.A.C., Murthy, S., Diaz, J. V., Slutsky, A.S., Villar, J., Angus, D.C., et al. (2020). Association between Administration of Systemic Corticosteroids and Mortality among Critically Ill Patients with COVID19: A Meta-analysis. JAMA - J. Am. Med. Assoc. 324 : 1330-1341.

Tang, W., Cao, Z., Han, M., Wang, Z., Chen, J., Sun, W., et al. (2020). Hydroxychloroquine in patients with mainly mild to moderate coronavirus disease 2019: Open label, randomised controlled trial. BMJ 369 $\therefore$

The Florentine (2020). Covid-19: Monoclonal antibody treatment developed in Siena.

Tiberghien, P., Lamballerie, X. de, Morel, P., Gallian, P., Lacombe, K., and Yazdanpanah, Y. (2020). Collecting and evaluating convalescent plasma for COVID-19 treatment: why and how? Vox Sang. 115 : $488-494$.

Tirupakuzhi Vijayaraghavan, B.K., Jha, V., Rajbhandari, D., Myatra, S.N., John, O., Ghosh, A., et al. (2020). Hydroxychloroquine plus personal protective equipment versus standard personal protective equipment alone for the prevention of COVID-19 infections among frontline healthcare workers: The HydrOxychloroquine Prophylaxis Evaluation(HOPE) trial: A structured summary of a study protocol for a randomized controlled trial. Trials $21: 754$. 
Tleyjeh, I.M., Kashour, Z., Damlaj, M., Riaz, M., Tlayjeh, H., Altannir, M., et al. (2020). Efficacy and safety of tocilizumab in COVID-19 patients: A living systematic review and meta-analysis. Clin. Microbiol. Infect.

Tomazini, B.M., Maia, I.S., Cavalcanti, A.B., Berwanger, O., Rosa, R.G., Veiga, V.C., et al. (2020). Effect of Dexamethasone on Days Alive and Ventilator-Free in Patients with Moderate or Severe Acute Respiratory Distress Syndrome and COVID-19: The CoDEX Randomized Clinical Trial. JAMA - J. Am. Med. Assoc. 324: 1307-1316.

University of Oxford (2020a). Aspirin to be investigated as a possible treatment for COVID-19 in the RECOVERY trial - RECOVERY Trial.

University of Oxford (2020b). Welcome - RECOVERY Trial.

US Food and Drug Administration (2020a). FDA cautions against use of hydroxychloroquine or chloroquine for COVID-19 outside of the hospital setting or a clinical trial due to risk of heart rhythm problems | FDA.

US Food and Drug Administration (2020b). Recommendations for Investigational COVID-19 Convalescent Plasma | FDA.

Valle, D.M. Del, Kim-Schulze, S., Huang, H.H., Beckmann, N.D., Nirenberg, S., Wang, B., et al. (2020). An inflammatory cytokine signature predicts COVID-19 severity and survival. Nat. Med. 26 : 1636-1643.

Voysey, M., Ann, S., Clemens, C., Madhi, S.A., Weckx, L.Y., Folegatti, P.M., et al. (2020). Articles Safety and efficacy of the ChAdOx1 nCoV-19 vaccine (AZD1222) against SARS-CoV-2: an interim analysis of four randomised controlled trials in Brazil, South Africa, and the UK. Lancet 0 :

Wang, J., Nikrad, M.P., Phang, T., Gao, B., Alford, T., Ito, Y., et al. (2011). Innate immune response to influenza A virus in differentiated human alveolar type II cells. Am. J. Respir. Cell Mol. Biol. 45 : $582-591$.

Wang, M., Wu, T., Zuo, Z., You, Y., Yang, X., Pan, L., et al. (2020a). Evaluation of current medical approaches for COVID-19: a systematic review and meta-analysis. BMJ Support. Palliat. Care bmjspcare2020-002554.

Wang, Y., Zhang, D., Du, G., Du, R., Zhao, J., Jin, Y., et al. (2020b). Remdesivir in adults with severe COVID-19: a randomised, double-blind, placebo-controlled, multicentre trial. Lancet 395 : 1569-1578.

WATANABE, S., CHAN, M., SUZUKI, W., and Nikkei staff writers (2020). China says Japan-developed drug Avigan works against coronavirus - Nikkei Asia.

Wit, E. de, Feldmann, F., Cronin, J., Jordan, R., Okumura, A., Thomas, T., et al. (2020). Prophylactic and therapeutic remdesivir (GS-5734) treatment in the rhesus macaque model of MERS-CoV infection. Proc. Natl. Acad. Sci. U. S. A. 117 : 6771-6776.

World Health Organization WHO recommends against the use of remdesivir in COVID-19 patients.

World Health Organization (2020a). Clinical management of COVID-19.

World Health Organization (2020b). Draft landscape of COVID-19 candidate vaccines.

World Health Organization (2020c). Solidarity clinical trial for COVID-19 treatments .

World Health Organization (2020d). WHO/Europe | Antimicrobial resistance - Preventing the COVID-19 pandemic from causing an antibiotic resistance catastrophe.

World Health Organization (2020e). WHO discontinues hydroxychloroquine and lopinavir/ritonavir treatment arms for COVID-19.

Worldometer (2020). Coronavirus Update (Live): 60,272,757 Cases and 1,418,260 Deaths from COVID-19 Virus Pandemic - Worldometer. 
Wu, X., Yu, K., Wang, Y., Xu, W., Ma, H., Hou, Y., et al. (2020). Efficacy and Safety of Triazavirin Therapy for Coronavirus Disease 2019: A Pilot Randomized Controlled Trial. Engineering.

Xu, X., Han, M., Li, T., Sun, W., Wang, D., Fu, B., et al. (2020). Effective treatment of severe COVID-19 patients with tocilizumab. Proc. Natl. Acad. Sci. U. S. A. 117 : 10970-10975.

Zemb, P., Bergman, P., Camargo, C.A., Cavalier, E., Cormier, C., Courbebaisse, M., et al. (2020). Vitamin D deficiency and the COVID-19 pandemic. J. Glob. Antimicrob. Resist. 22 : 133-134.

Zhang, B., Liu, S., Tan, T., Huang, W., Dong, Y., Chen, L., et al. (2020a). Treatment With Convalescent Plasma for Critically Ill Patients With Severe Acute Respiratory Syndrome Coronavirus 2 Infection. Chest158: e9-e13.

Zhang, X., Zhang, Y., Qiao, W., Zhang, J., and Qi, Z. (2020b). Baricitinib, a drug with potential effect to prevent SARS-COV-2 from entering target cells and control cytokine storm induced by COVID-19. Int. Immunopharmacol. 86 :.

Zhang, Z., Wang, S., Tu, X., Peng, X., Huang, Y., Wang, L., et al. (2020c). A comparative study on the time to achieve negative nucleic acid testing and hospital stays between danoprevir and lopinavir/ritonavir in the treatment of patients with COVID-19. J. Med. Virol. 92 : 2631-2636.

Zhu, Z., Lu, Z., Xu, T., Chen, C., Yang, G., Zha, T., et al. (2020). Arbidol monotherapy is superior to lopinavir/ritonavir in treating COVID-19. J. Infect. 81 : e21-e23. 